\title{
Integral Conditions for Convergence of Solutions of Non-Linear Robin's Problem in Strongly Perforated Domain
}

\author{
E.Ya. Khruslov ${ }^{1}$, L.O. Khilkova ${ }^{2}$, and M.V. Goncharenko ${ }^{3}$ \\ ${ }^{1,3}$ B. Verkin Institute for Low Temperature Physics and Engineering \\ of the National Academy of Sciences of Ukraine \\ 47 Nauky Ave., Kharkiv 61103, Ukraine \\ E-mail: ${ }^{1}$ khruslov@ilt.kharkov.ua \\ ${ }^{3}$ marusya61@yahoo.co.uk \\ ${ }^{2}$ Institute of Chemical Technologies \\ of Volodymyr Dahl East Ukrainian National University \\ 31 Volodymyrska Str., Rubizhne 93009, Ukraine \\ E-mail: ${ }^{2}$ LarisaHilkova@gmail.com
}

Received May 27, 2017

\begin{abstract}
We consider a boundary-value problem for the Poisson equation in a strongly perforated domain $\Omega^{\varepsilon}=\Omega \backslash F^{\varepsilon} \subset R^{n}(n \geqslant 2)$ with non-linear Robin's condition on the boundary of the perforating set $F^{\varepsilon}$. The domain $\Omega^{\varepsilon}$ depends on the small parameter $\varepsilon>0$ such that the set $F^{\varepsilon}$ becomes more and more loosened and distributes more densely in the domain $\Omega$ as $\varepsilon \rightarrow 0$. We study the asymptotic behavior of the solution $u^{\varepsilon}(x)$ of the problem as $\varepsilon \rightarrow 0$. A homogenized equation for the main term $u(x)$ of the asymptotics of $u^{\varepsilon}(x)$ is constructed and the integral conditions for the convergence of $u^{\varepsilon}(x)$ to $u(x)$ are formulated.
\end{abstract}

Key words: homogenization, stationary diffusion, non-linear Robin's boundary condition, homogenized equation.

Mathematical Subject Classification 2010: 35Q70.

\section{Introduction}

Let $\Omega$ be a fixed bounded domain in $R^{n}(n \geqslant 2)$ and $F^{\varepsilon}$ be a closed set in $\Omega$, depending on a small parameter $\varepsilon$ so that, as $\varepsilon \rightarrow 0$, the set $F^{\varepsilon}$ becomes more and more loosened and distributes more densely in $\Omega$. We call $F^{\varepsilon}$ the perforating set. Denote by $\Omega^{\varepsilon}=\Omega \backslash F^{\varepsilon}$ a strongly perforated domain. The boundary of the 
domain $\Omega^{\varepsilon}$ consists of two disjoint parts: $\partial \Omega^{\varepsilon}=\partial \Omega \cup \partial F^{\varepsilon}$, where $\partial \Omega$ is the outer boundary, $\partial F^{\varepsilon}$ is the boundary of the perforating set $F^{\varepsilon}$. We assume that these boundaries are smooth.

In the domain $\Omega^{\varepsilon}$ we consider a boundary-value problem

$$
\left\{\begin{array}{l}
-\Delta u^{\varepsilon}=f^{\varepsilon}(x), \quad x \in \Omega^{\varepsilon}, \\
\frac{\partial u^{\varepsilon}}{\partial \nu}+\sigma^{\varepsilon}\left(x, u^{\varepsilon}\right)=0, \quad x \in \partial F^{\varepsilon}, \\
u^{\varepsilon}=0 \text { on } \partial \Omega,
\end{array}\right.
$$

where $\Delta$ is the Laplacian, $\nu$ is the unit outer normal to the boundary $\partial F^{\varepsilon}$ with respect to the domain $\Omega^{\varepsilon}$, the functions $f^{\varepsilon}(x): \Omega^{\varepsilon} \rightarrow R^{1}$ and $\sigma^{\varepsilon}(x, u): \Omega^{\varepsilon} \times$ $R^{1} \rightarrow R^{1}$ are given.

Problem (1) describes the process of stationary diffusion in a porous medium $\Omega^{\varepsilon}$. The function $u^{\varepsilon}(x)$ is a concentration of a diffusing substance.

The geometry of the domain $\Omega^{\varepsilon}$ is very complicated. It is practically impossible to solve problem (1) either by analytical or numerical methods. In the case under consideration, the scale $\varepsilon$ of the microstructure of the medium is much smaller than the scale of the physical process, therefore we can proceed to study the asymptotic behavior of the solution $u^{\varepsilon}(x)$ as $\varepsilon \rightarrow 0$. As a result, we obtain the homogenized equation. The corresponding homogenized problem is a macroscopic model of the process.

The asymptotic analysis of problems similar to problem (1) was carried out by many authors (see, for example, $[1-7,14,16,17])$. Note that in all these papers the domains $\Omega^{\varepsilon}=\Omega \backslash F^{\varepsilon}$ are of special type. Namely, the perforating set $F^{\varepsilon}$ is a union of periodically located (with a period $\varepsilon$ ) identical bodies of a diameter $O(\varepsilon)$.

However, the domains $\Omega^{\varepsilon}$ of an arbitrary form (including the connected perforating sets $F^{\varepsilon}$ ) are more natural from the physical point view. Earlier, in [9] we considered problem (1) in the domains $\Omega^{\varepsilon}$ of an arbitrary form satisfying only one important condition: the condition of strong connectedness (this condition was introduced in [12]). It was shown that the solution $u^{\varepsilon}(x)$ of problem (1) converges as $\varepsilon \rightarrow 0$ in the $L^{2}\left(\Omega^{\varepsilon}\right)$ metric to the solution $u(x)$ of the homogenized problem in the domain $\Omega$ :

$$
-\sum_{i, k=1}^{n} \frac{\partial}{\partial x_{i}}\left(a_{i k}(x) \frac{\partial u}{\partial x_{k}}\right)+\frac{1}{2} c_{u}(x, u)=f(x), x \in \Omega, \quad u(x)=0, x \in \partial \Omega .
$$

Here $\left\{a_{i j}\right\}_{i, j=1}^{n}$ is the positive-definite tensor characterizing the effective conductivity of the porous medium, $c_{u}(x, u)=\frac{\partial}{\partial u} c(x, u)$, and the function $c(x, u)$ characterizes the effective absorption properties of the medium. They are determined 
by the so-called mesoscopic characteristics of the domains $\Omega^{\varepsilon}$ ( [12]). These characteristics are introduced in cubes ("mesocubes"), which are small relative to the whole domain but at the same time are large relative to the microscale. The structure of the strongly connected domain $\Omega^{\varepsilon}$ and the absorption on the boundary in the cube we characterize with the help of the mesoscopic characteristic $a(x, \varepsilon, h)$. It can be the mean conductivity tensor or the absorption function.

In [9], it is assumed that uniformly with respect to $x \in \Omega$ there exists a density of mesoscopic characteristic:

$$
\lim _{h \rightarrow 0} \varlimsup_{\varepsilon \rightarrow 0} \frac{a(x, \varepsilon, h)}{h^{n}}=\lim _{h \rightarrow 0} \lim _{\varepsilon \rightarrow 0} \frac{a(x, \varepsilon, h)}{h^{n}}=a(x) .
$$

The authors proved that under these conditions the solution of problem (1) converges in $L^{2}\left(\Omega^{\varepsilon}\right)$ to the solution $u(x)$ of homogenized problem (2).

In the present paper, we obtain a similar result, but under weaker integral conditions

$$
\lim _{h \rightarrow 0} \varlimsup_{\varepsilon \rightarrow 0} \int_{\Omega}\left|\frac{a(x, \varepsilon, h)}{h^{n}}-a(x)\right| d x=0
$$

imposed on the mesoscopic characteristics $a(x, \varepsilon, h)$. We prove that this condition is sufficient, but it is very probable that it is also necessary for the convergence of $u^{\varepsilon}(x)$ to $u(x)$.

The paper is organized as follows. In Section 2, we formulate the problem and explain the main result. Section 3 contains the proof of the existence and uniqueness theorem for the weak solution of problem (1) for each fixed $\varepsilon$. In Section 4 we state auxiliary lemmas. In Section 5 we prove the main theorem. This section is divided into several subsections correspondingly to the major steps in our proof.

\section{Statement of the Problem and Main Result}

Let $\Omega$ be a bounded domain in $R^{n}(n \geqslant 2)$, and $F^{\varepsilon}$ be a closed set in $\Omega$ with smooth boundary. We assume that the domains $\Omega^{\varepsilon}=\Omega \backslash F^{\varepsilon}$ satisfy the following conditions:

1) for any $\varepsilon>0$ for any subdomain $\Omega^{\prime} \subset \Omega$,

$$
\operatorname{mes}\left\{\Omega^{\varepsilon} \cap \Omega^{\prime}\right\} \geqslant C_{1} \cdot \operatorname{mes}\left\{\Omega^{\prime}\right\}>0 ;
$$

2) the domains $\Omega^{\varepsilon}$ satisfy the extension condition, i.e., for any function $\vartheta^{\varepsilon}(x) \in$ $H^{1}\left(\Omega^{\varepsilon}\right)$, there exists a function $\widetilde{\vartheta}^{\varepsilon}(x) \in H^{1}(\Omega)$ such that $\vartheta^{\varepsilon}(x)=\widetilde{\vartheta}^{\varepsilon}(x)$ for $x \in \Omega^{\varepsilon}$, and the inequality

$$
\left\|\nabla \widetilde{\vartheta}^{\varepsilon}\right\|_{L^{2}(\Omega)} \leqslant C_{2}\left\|\nabla \vartheta^{\varepsilon}\right\|_{L^{2}\left(\Omega^{\varepsilon}\right)}
$$

is true. Here the constants $C_{1}, C_{2}$ are independent of $\varepsilon$. 
Remark 1. From conditions (4), (5) it follows that the inequality

$$
\left\|\widetilde{\vartheta}^{\varepsilon}\right\|_{H^{1}(\Omega)} \leqslant C\left\|\vartheta^{\varepsilon}\right\|_{H^{1}\left(\Omega^{\varepsilon}\right)}
$$

is correct, where the constant $C$ is independent of $\varepsilon$. And, therefore, the domains $\Omega^{\varepsilon}$ satisfy the condition of strong connectivity (see $[12, \S 4.2]$ ).

In the domain $\Omega^{\varepsilon}=\Omega \backslash F^{\varepsilon}$, we consider boundary-value problem (1) where the functions $f^{\varepsilon}(x) \in L^{2}\left(\Omega^{\varepsilon}\right)$ and $\sigma^{\varepsilon}(x, u) \in C\left(\Omega \times R^{1}\right)$ are given. Assume that for any $\varepsilon$ the function $\sigma^{\varepsilon}(x, u)$ satisfies the following conditions:

$a_{1}: \forall u_{1}, u_{2} \in R^{1}:\left(\sigma^{\varepsilon}\left(x, u_{1}\right)-\sigma^{\varepsilon}\left(x, u_{2}\right)\right) \cdot\left(u_{1}-u_{2}\right) \geqslant 0$;

$a_{2}: \sigma^{\varepsilon}(x, 0)=0$;

$a_{3}: \forall u \in R^{1}:\left|\sigma^{\varepsilon}(x, u)\right| \leqslant \widehat{\sigma}^{\varepsilon}(x) \cdot\left(1+|u|^{\Theta}\right),\left(\Theta<\frac{n}{n-2}\right)$. Here the function $\widehat{\sigma}^{\varepsilon}(x) \in C(\Omega), \widehat{\sigma}^{\varepsilon}(x) \geqslant 0$, and for any ball $B(\rho, z)$ of radius $\rho(0<\rho<1)$ centered at the point $z \in \Omega$,

$$
\int_{\partial F^{\varepsilon} \cap B(\rho, z)} \widehat{\sigma}^{\varepsilon}(x) d \Gamma<C_{1} \rho^{n}+C_{2}(\varepsilon) \rho^{n-1},
$$

where the constants $C_{1}, C_{2}$ are independent of $z, \rho, C_{1}$ is independent of $\varepsilon$, and $C_{2}(\varepsilon) \rightarrow 0$ as $\varepsilon \rightarrow 0$.

We denote

$$
H^{1}\left(\Omega^{\varepsilon}, \partial \Omega\right)=\left\{u(x) \in H^{1}\left(\Omega^{\varepsilon}\right):\left.u\right|_{\partial \Omega}=0\right\} .
$$

Definition 1. A generalized solution of problem (1) is a function $u^{\varepsilon}(x) \in$ $H^{1}\left(\Omega^{\varepsilon}, \partial \Omega\right)$ satisfying the identity

$$
\int_{\Omega^{\varepsilon}}\left(\nabla u^{\varepsilon}, \nabla \varphi\right) d x+\int_{\partial F^{\varepsilon}} \sigma^{\varepsilon}\left(x, u^{\varepsilon}\right) \varphi d \Gamma=\int_{\Omega^{\varepsilon}} f^{\varepsilon} \varphi d x, \quad \forall \varphi(x) \in H^{1}\left(\Omega^{\varepsilon}, \partial \Omega\right) .
$$

Theorem 1. Problem (1) has the unique generalized solution $u^{\varepsilon}(x) \in$ $H^{1}\left(\Omega^{\varepsilon}, \partial \Omega\right)$ for each fixed $\varepsilon$.

The proof of this theorem is given in Section 3

The main goal of this paper is to study the asymptotic behavior of $u^{\varepsilon}(x)$ as $\varepsilon \rightarrow 0$. To this end, we first define in what sense we mean the convergence of the solutions $u^{\varepsilon}(x)$ and introduce the "mesoscopic" characteristics of the domain $\Omega^{\varepsilon}$.

Definition 2. We say that a sequence of functions $\left\{u^{\varepsilon}(x)\right\}_{\varepsilon} \in L^{p}\left(\Omega^{\varepsilon}\right)$ converges in $L^{p}\left(\Omega^{\varepsilon}, \Omega\right)$ if there exists a function $u(x) \in L^{p}(\Omega)$ such that

$$
\lim _{\varepsilon \rightarrow 0}\left\|u^{\varepsilon}-\chi^{\varepsilon} u\right\|_{L^{p}\left(\Omega^{\varepsilon}\right)}=0,
$$

where $\chi^{\varepsilon}(x)$ is a characteristic function of the domain $\Omega^{\varepsilon}$. 
Let us introduce the "mesoscopic" characteristics of the domain $\Omega^{\varepsilon}$. These characteristics are the local characteristics of the microstructure considered in the cube $K_{h}^{z}=K(z, h)$ centered at the point $z$ with the edges of length $h$ oriented along the coordinate axes.

We define the quantitative characteristic of conductivity by the functional with respect to an arbitrary vector $\ell \in R^{n}$,

$$
T_{h, z}^{\varepsilon}(\ell)=\inf _{v^{\varepsilon}} \int_{K_{h}^{z} \cap \Omega^{\varepsilon}}\left(\left|\nabla v^{\varepsilon}\right|^{2}+h^{-2-\tau}\left|v^{\varepsilon}-(x-z, \ell)\right|^{2}\right) d x,
$$

where the lower bound is taken in a class of functions $v^{\varepsilon}(x) \in H^{1}\left(K_{h}^{z} \cap \Omega^{\varepsilon}\right), \tau \in$ $(0,2)$ is the parameter of penalty. For any $\ell \in R^{n}$, there exists a unique minimizer of the functional (8) that can be represented in the form (see [12, p. 4, §1])

$$
v^{\varepsilon}=\sum_{i=1}^{n} v_{i}^{\varepsilon} \ell^{i} .
$$

The function $v_{i}^{\varepsilon}$ minimizes (8) for $\ell=e^{i}\left(e^{i}\right.$ is a basic vector of the axis $x_{i}$ ). This implies that functional (8) is homogeneously quadratic with respect to $\ell$, i.e.,

$$
T_{h, z}^{\varepsilon}(\ell)=\sum_{i, j=1}^{n} a_{i j}(z, \varepsilon, h) \ell_{i} \ell_{j},
$$

where the coefficients $a_{i j}(z, \varepsilon, h)$ are defined by

$$
\begin{aligned}
& a_{i j}(z, \varepsilon, h) \\
& \quad=\int_{K_{h}^{z} \cap \Omega^{\varepsilon}}\left\{\left(\nabla v_{i}^{\varepsilon}, \nabla v_{j}^{\varepsilon}\right)+h^{-2-\tau}\left[v_{i}^{\varepsilon}-\left(x_{i}-z_{i}\right)\right]\left[v_{j}^{\varepsilon}-\left(x_{j}-z_{j}\right)\right]\right\} d x .
\end{aligned}
$$

The system of numbers $\left\{a_{i j}(z, \varepsilon, h)\right\}_{i, j=1}^{n}$ forms a symmetric positive definite tensor in $R^{n}$. The tensor characterizes the conductivity of the domains $\Omega^{\varepsilon}$ in the cube $K_{h}^{z}$.

We define the quantitative characteristic of absorption on the boundary $\partial F^{\varepsilon}$ by the functional for arbitrary $s \in R^{1}$,

$$
\begin{aligned}
& c(z, s ; \varepsilon, h) \\
& =\inf _{w^{\varepsilon}}\left[\int_{K_{h}^{z} \cap \Omega^{\varepsilon}}\left\{\left|\nabla w^{\varepsilon}\right|^{2}+h^{-2-\tau}\left|w^{\varepsilon}-s\right|^{2}\right\} d x+\int_{K_{h}^{z} \cap \partial F^{\varepsilon}} g^{\varepsilon}\left(x, w^{\varepsilon}\right) d \Gamma\right],
\end{aligned}
$$

where the infimum is taken in a class of functions $w^{\varepsilon} \in H^{1}\left(K_{h}^{z} \cap \Omega^{\varepsilon}\right), \tau \in(0,2)$ is the parameter of penalty, and the function $g^{\varepsilon}(x, u)$ is defined by

$$
g^{\varepsilon}(x, u):=2 \int_{0}^{u} \sigma^{\varepsilon}(x, s) d s .
$$


It can be shown that for any fixed $\varepsilon, h, s\left(0<\varepsilon \ll h \ll 1, C_{1}<s<C_{2}\right)$, the functions $\frac{a_{i j}(X, \varepsilon, h)}{h^{n}}$ and $\frac{c(x, s, \varepsilon, h)}{h^{n}}$ are measurable and bounded functions of $x$.

The main result of this paper is the following theorem.

Theorem 2. Let the domains $\Omega^{\varepsilon}$ be strongly connected, the function $\sigma^{\varepsilon}(x, u)$ satisfy the conditions $a_{1}-a_{3}$ and there exist $\tau \in(0,2)$ for which the functions $a_{i j}(x, \varepsilon, h)(i, j=\overline{1, n}), c(x, s ; \varepsilon, h)$

1) $\lim _{h \rightarrow 0} \varlimsup_{\varepsilon \rightarrow 0} \int_{\Omega}\left|\frac{a_{i j}(x, \varepsilon, h)}{h^{n}}-a_{i j}(x)\right| d x=0$,

where $a_{i j}(x)$ are piecewise continuous functions of $x$, and $\left\{a_{i j}(x)\right\}_{i, j=1}^{n}$ is a symmetric positive definite tensor in $R^{n}$;

2) $\lim _{h \rightarrow 0} \varlimsup_{\varepsilon \rightarrow 0} \int_{\Omega}\left|\frac{c(x, s, \varepsilon, h)}{h^{n}}-c(x, s)\right| d x=0, \forall s \in R^{1}$,

where the function $c(x, s)$ is bounded in $x$ and differentiable with respect to $s$, and its derivative $c_{s}(x, s)=\frac{\partial}{\partial s} c(x, s)$ satisfies the conditions:

$$
\begin{array}{ll}
\forall s_{1}, s_{2} \in R^{1}: \quad\left(c_{s}\left(x, s_{1}\right)-c_{s}\left(x, s_{2}\right)\right)\left(s_{1}-s_{2}\right) \geqslant 0, \\
\forall s \in R^{1}: & c_{s}(x, s) \leqslant C\left(1+|s|^{\Theta}\right), \quad\left(\Theta<\frac{n+2}{n-2}\right) ;
\end{array}
$$

3) the functions $f^{\varepsilon}(x)$ extended by zero to $F^{\varepsilon}$ converge weakly in $L^{2}(\Omega)$ to the function $f(x)$.

Then the generalized solution $u^{\varepsilon}(x)$ of problem (1) converges in $L^{p}\left(\Omega^{\varepsilon}, \Omega\right)$ $\left(p<\frac{2 n}{n-2}\right)$ to the function $u(x)$, which is a generalized solution of homogenization problem (2).

We recall that a generalized solution of homogenized problem (2) is a function $u(x)$ from the space $\stackrel{H}{H}(\Omega)$ satisfying the identity

$$
\int_{\Omega} \sum_{i, j=1}^{n} a_{i j} \frac{\partial u}{\partial x_{i}} \frac{\partial \varphi}{\partial x_{j}} d x+\frac{1}{2} \int_{\Omega} c_{u}(x, u) \varphi d \Gamma=\int_{\Omega} f \varphi d x, \quad \forall \varphi(x) \in \stackrel{\circ}{H}^{1}(\Omega) .
$$

Remark 2. Since the mesoscopic characteristics $a_{i k}(x, \varepsilon, h)$ and $c(x, s ; \varepsilon, h)$ depend on the parameter of penalty $\tau$, the limit functions $a_{i k}(x)$ and $c(x, s)$ must also depend on $\tau$ formally. However, if conditions 1) and 2) of Theorem 2 are satisfied for some $\tau \in(0,2)$, then the solution $u^{\varepsilon}(x)$ of problem (1) converges to the solution $u(x)$ of limit problem (2) for any right-hand side $f(x)$. This solution does not have to depend on $\tau$ as a limit of the original solutions $u^{\varepsilon}(x)$ is independent of $\tau$. Taking this fact into account, it can be shown that the limit coefficients $a_{i k}(x)$ and $c(x, s)$ are also independent of $\tau$. 
Let us give the simplest example, where conditions (3) are not satisfied uniformly, but the integral conditions are satisfied.

Let $\Omega$ be a bounded domain in $R^{3}$, and $G$ be a subdomain compactly embedded in the domain $\Omega$. We assume that the set $F^{\varepsilon}=\bigcup_{i} F_{i}^{\varepsilon}$ is periodically distributed in subdomain $G$. The set $F^{\varepsilon}$ consists of the sets $F_{i}^{\varepsilon}=\varepsilon F+x^{i \varepsilon}\left(x^{i \varepsilon} \in\right.$ $\left.\varepsilon \mathbb{Z}^{n}\right)$ that are a contraction of some fixed domain $F \in K_{1}$ with smooth boundary $\partial F$ ( $K_{1}$ is a unit cube). In the domain $\Omega^{\varepsilon}=\Omega \backslash F^{\varepsilon}$, we consider boundary-value problem (1) for $\sigma^{\varepsilon}(x, u)=\varepsilon \cdot \sigma(x, u)$ where $\sigma(x, u) \in C\left(\Omega \times R^{1}\right)$.

Conditions (3) for this problem do not hold uniformly at the points of $\Omega$, but the integral conditions are satisfied. And the limit characteristics of the domains $\Omega^{\varepsilon}$ are calculated by the formulas:

$$
\begin{gathered}
c(x, u)=2|\partial F| \chi(x) \int_{0}^{u} \sigma(x, r) d r \\
a_{i j}(x)=\delta_{i j}-\chi(x)\left[\delta_{i j}|F|+\int_{K_{1} \backslash F} \sum_{k=1}^{n} \frac{\partial V_{i}(\xi)}{\partial \xi_{k}} \frac{\partial V_{j}(\xi)}{\partial \xi_{k}} d \xi\right], \quad i, j=1, \ldots, n .
\end{gathered}
$$

Here $|\partial F|,|F|$ are an area of the surface $\partial F$ and a volume of the domain $F$, respectively, $\chi(x)$ is a characteristic function of the domain $G$, the function $V_{j}(\xi)(j=$ $\overline{1, n})$ is a solution of the "cell" problem in the unit cube $K_{1}$ :

$$
\begin{aligned}
& \sum_{i=1}^{n} \frac{\partial^{2} V_{j}(\xi)}{\partial \xi_{i}^{2}}=0, \quad \xi \in K_{1} \backslash F, \\
& \frac{\partial V_{j}(\xi)}{\partial \nu_{\xi}}=\cos \left(\nu(\xi), e^{j}\right), \quad \xi \in \partial F, \\
& \left.V_{j}\right|_{\Gamma_{i}^{+}}=\left.V_{j}\right|_{\Gamma_{i}^{-}},\left.\quad \frac{\partial V_{j}}{\partial \xi_{i}}\right|_{\Gamma_{i}^{+}}=\left.\frac{\partial V_{j}}{\partial \xi_{i}}\right|_{\Gamma_{i}^{-}}, \quad i=\overline{1, n}, \\
& \int_{K_{1} \backslash F} V_{j}(\xi) d \xi=0,
\end{aligned}
$$

here $\Gamma_{i}^{ \pm}$are opposite sides of the cube $K_{1}, \nu=\nu(\xi)$ is a unit normal with respect to the boundary of the domain $F$.

For more general case, we refer the reader to [10].

\section{Proof of Theorem 1}

Let us prove the existence and the uniqueness of the generalized solution of problem (1) for each fixed $\varepsilon$. In the proof we will follow the method developed in $[8]$. 
The solution of problem (1) minimizes the functional

$$
\Phi^{\varepsilon}\left[u^{\varepsilon}\right]=\int_{\Omega^{\varepsilon}}\left|\nabla u^{\varepsilon}\right|^{2} d x+\int_{\partial F^{\varepsilon}} g^{\varepsilon}\left(x, u^{\varepsilon}\right) d \Gamma-2 \int_{\Omega^{\varepsilon}} f^{\varepsilon} u^{\varepsilon} d x
$$

on the class of functions $u^{\varepsilon}(x) \in H^{1}\left(\Omega^{\varepsilon}, \partial \Omega\right)$. Here $g^{\varepsilon}\left(x, u^{\varepsilon}\right)$ is defined by (13).

According to [8], the existence of a minimizer of the functional (15) follows from the coercivity and the weak lower semicontinuity of the functional.

Let us show that the functional $\Phi^{\varepsilon}[\cdot]$ is coercive. By the extension condition (5) and the Friedrichs inequality, we get

$$
\left\|u^{\varepsilon}\right\|_{L^{2}\left(\Omega^{\varepsilon}\right)} \leqslant\left\|\widetilde{u}^{\varepsilon}\right\|_{L^{2}(\Omega)} \leqslant C_{1}\left\|\nabla \widetilde{u}^{\varepsilon}\right\|_{L^{2}(\Omega)} \leqslant C_{2}\left\|\nabla u^{\varepsilon}\right\|_{L^{2}\left(\Omega^{\varepsilon}\right)},
$$

where the constants $C_{1}, C_{2}$ are independent of $\varepsilon$. Here the function $\widetilde{u}^{\varepsilon}(x)$ is an extension of the function $u^{\varepsilon}(x)$ on the whole domain $\Omega$. Further, by the Young and the Cauchy-Bunyakovski inequalities, taking into account that $g^{\varepsilon}(x, u) \geqslant 0$, we obtain

$$
\begin{aligned}
\Phi^{\varepsilon}\left[u^{\varepsilon}\right] & =\int_{\Omega^{\varepsilon}}\left|\nabla u^{\varepsilon}\right|^{2} d x+\int_{\partial F^{\varepsilon}} g^{\varepsilon}\left(x, u^{\varepsilon}\right) d \Gamma-2 \int_{\Omega^{\varepsilon}} f^{\varepsilon} u^{\varepsilon} d x \\
& \geqslant \int_{\Omega^{\varepsilon}}\left|\nabla u^{\varepsilon}\right|^{2} d x-2\left|\int_{\Omega^{\varepsilon}} f^{\varepsilon} u^{\varepsilon} d x\right| \\
& \geqslant \frac{1}{2} \int_{\Omega^{\varepsilon}}\left|\nabla u^{\varepsilon}\right|^{2} d x+\frac{1}{2} \int_{\Omega^{\varepsilon}}\left|\nabla u^{\varepsilon}\right|^{2} d x-\frac{1}{2 \delta} \int_{\Omega^{\varepsilon}}\left|u^{\varepsilon}\right|^{2} d x-2 \delta \int_{\Omega^{\varepsilon}}\left|f^{\varepsilon}\right|^{2} d x \\
& \geqslant \frac{1}{2} \int_{\Omega^{\varepsilon}}\left|\nabla u^{\varepsilon}\right|^{2} d x+\left(\frac{1}{2 C}-\frac{1}{2 \delta}\right) \int_{\Omega^{\varepsilon}}\left|u^{\varepsilon}\right|^{2} d x-2 \delta\left\|f^{\varepsilon}\right\|_{L^{2}\left(\Omega^{\varepsilon}\right)}^{2} .
\end{aligned}
$$

Setting $C_{1}=\min \left(\frac{1}{2}, \frac{1}{4 C}\right)$ and $C_{2}=4 C\left\|f^{\varepsilon}\right\|_{L^{2}\left(\Omega^{\varepsilon}\right)}^{2}$, we get the inequality

$$
\Phi^{\varepsilon}[u] \geqslant C_{1}\|u\|_{H^{1}\left(\Omega^{\varepsilon}\right)}^{2}-C_{2}
$$

for any function $u \in H^{1}\left(\Omega^{\varepsilon}, \partial \Omega\right)$. It establishes that the functional $\Phi^{\varepsilon}[\cdot]$ is coercive in the space $H^{1}\left(\Omega^{\varepsilon}, \partial \Omega\right)$.

It remains to prove that the functional $\Phi^{\varepsilon}[\cdot]$ is weakly lower semicontinuous. We now proceed analogously to [14].

We write the functional $\Phi^{\varepsilon}[\cdot]$ in the form

$$
\Phi^{\varepsilon}\left[u^{\varepsilon}\right]=\int_{\Omega^{\varepsilon}} L\left(\nabla u^{\varepsilon}, u^{\varepsilon}, x\right) d x,
$$

where $L(p, u, x)$ is the Lagrangian of the functional. To do this, we need the following lemma. 
Lemma 1. For any function $\varphi \in H^{1}\left(\Omega^{\varepsilon}, \partial \Omega\right)$, the equality

$$
\int_{\partial F^{\varepsilon}} \varphi d \Gamma=\int_{\Omega^{\varepsilon}}\left(\nabla \psi_{0}, \nabla \varphi\right) d x-\frac{\left|\partial F^{\varepsilon}\right|}{\left|\Omega^{\varepsilon}\right|} \int_{\Omega^{\varepsilon}} \varphi d x
$$

holds, where $\left|\partial F^{\varepsilon}\right|,\left|\Omega^{\varepsilon}\right|$ are the surface and volume measures of the corresponding sets, and the function $\psi_{0}(x)$ is a solution of the boundary-value problem

$$
\left\{\begin{array}{l}
-\Delta \psi_{0}=\frac{\left|\partial F^{\varepsilon}\right|}{\left|\Omega^{\varepsilon}\right|}, \quad x \in R^{n} \backslash F^{\varepsilon}, \\
\frac{\partial \psi_{0}}{\partial \nu}=1, \quad x \in \partial F^{\varepsilon} \\
\psi_{0}(x) \rightarrow 0 \quad \text { as } \quad|x| \rightarrow \infty
\end{array}\right.
$$

Proof. The existence and the uniqueness of the solution $\psi_{0}(x)$ can be proved by the standard methods of potential theory ( [15, Chap. 4]), taking into account the smoothness of the boundary $\partial F^{\varepsilon}$.

We multiply the differential equation of the problem by an arbitrary function $\varphi \in H^{1}\left(\Omega^{\varepsilon}, \partial \Omega\right)$ and integrate over the domain $\Omega^{\varepsilon}$. Using the integration by parts and the boundary conditions, we obtain the required equality (16).

With the help of Lemma 1 we write the functional $\Phi^{\varepsilon}[\cdot]$ in the form

$$
\begin{aligned}
\Phi^{\varepsilon}\left[u^{\varepsilon}\right]= & \int_{\Omega^{\varepsilon}}\left|\nabla u^{\varepsilon}\right|^{2} d x+\int_{\Omega^{\varepsilon}}\left(\nabla \psi_{0}, \nabla_{x} g^{\varepsilon}\left(x, u^{\varepsilon}\right)\right) d x \\
& -\frac{\left|\partial F^{\varepsilon}\right|}{\left|\Omega^{\varepsilon}\right|} \int_{\Omega^{\varepsilon}} g^{\varepsilon}\left(x, u^{\varepsilon}\right) d x-2 \int_{\Omega^{\varepsilon}} f^{\varepsilon} u^{\varepsilon} d x \\
= & \int_{\Omega^{\varepsilon}}\left|\nabla u^{\varepsilon}\right|^{2} d x+2 \int_{\Omega^{\varepsilon}} \int_{0}^{u^{\varepsilon}}\left(\nabla \psi_{0}, \nabla_{x} \sigma^{\varepsilon}(x, s)\right) d s d x \\
& +\int_{\Omega^{\varepsilon}}\left(\nabla \psi_{0}, \nabla u^{\varepsilon}\right) \cdot \sigma^{\varepsilon}\left(x, u^{\varepsilon}\right) d x \\
& \quad-\frac{\left|\partial F^{\varepsilon}\right|}{\left|\Omega^{\varepsilon}\right|} \int_{\Omega^{\varepsilon}} g^{\varepsilon}\left(x, u^{\varepsilon}\right) d x-2 \int_{\Omega^{\varepsilon}} f^{\varepsilon} u^{\varepsilon} d x=: \int_{\Omega^{\varepsilon}} L\left(\nabla u^{\varepsilon}, u^{\varepsilon}, x\right) d x .
\end{aligned}
$$

Let $p=\left(p_{1}, \ldots, p_{n}\right):=\nabla u^{\varepsilon}$,

$$
\begin{aligned}
L(p, u, x)=\sum_{i=1}^{n} p_{i}^{2} & +2 \int_{0}^{u}\left(\nabla \psi_{0}, \nabla_{x} \sigma^{\varepsilon}(x, s)\right) d s \\
& +\sigma^{\varepsilon}(x, u) \sum_{i=1}^{n} \frac{\partial \psi_{0}}{\partial x_{i}} \cdot p_{i}-\frac{\left|\partial F^{\varepsilon}\right|}{\left|\Omega^{\varepsilon}\right|} g^{\varepsilon}(x, u)-2 f^{\varepsilon} u .
\end{aligned}
$$

Journal of Mathematical Physics, Analysis, Geometry, 2017, Vol. 13, No. 3 
Since

$$
\sum_{i, j=1}^{n} \frac{\partial^{2} L}{\partial p_{i} \partial p_{j}} \eta_{i} \eta_{j}=2 \sum_{i=1}^{n} \eta_{i}^{2}=2|\eta|^{2}, \quad \forall p, \eta \in R^{n}, \quad x \in \Omega^{\varepsilon},
$$

the function $L$ is uniformly convex in $p$ for each $x \in \Omega^{\varepsilon}$. This means that the functional $\Phi^{\varepsilon}[\cdot]$ is weakly lower semicontinuous on $H^{1}\left(\Omega^{\varepsilon}, \partial \Omega\right)$.

Thus, the functional $\Phi^{\varepsilon}[\cdot]$ is coercive and weakly lower semicontinuous and there exists at least one minimizer (see [8, Sect. 8.2]). Therefore, there exists at least one generalized solution of problem (1). Our next goal is to prove that this solution is unique.

We assume that problem (1) has two generalized solution $u_{1}^{\varepsilon}(x), u_{2}^{\varepsilon}(x)$. Then, by (7) for any function $\varphi \in H^{1}\left(\Omega^{\varepsilon}, \partial \Omega\right)$, the identities

$$
\begin{aligned}
& \int_{\Omega^{\varepsilon}}\left(\nabla u_{1}^{\varepsilon}, \nabla \varphi\right) d x+\int_{\partial F^{\varepsilon}} \sigma^{\varepsilon}\left(x, u_{1}^{\varepsilon}\right) \varphi d \Gamma=\int_{\Omega^{\varepsilon}} f^{\varepsilon} \varphi d x \\
& \int_{\Omega^{\varepsilon}}\left(\nabla u_{2}^{\varepsilon}, \nabla \varphi\right) d x+\int_{\partial F^{\varepsilon}} \sigma^{\varepsilon}\left(x, u_{2}^{\varepsilon}\right) \varphi d \Gamma=\int_{\Omega^{\varepsilon}} f^{\varepsilon} \varphi d x
\end{aligned}
$$

are true. Subtracting (19) from (18) and taking $\varphi(x)=u_{1}^{\varepsilon}(x)-u_{2}^{\varepsilon}(x)$ as the test function, we obtain

$$
\int_{\Omega^{\varepsilon}}\left|\nabla u_{1}^{\varepsilon}-\nabla u_{2}^{\varepsilon}\right|^{2} d x+\int_{\partial F^{\varepsilon}}\left(\sigma^{\varepsilon}\left(x, u_{1}^{\varepsilon}\right)-\sigma^{\varepsilon}\left(x, u_{2}^{\varepsilon}\right)\right) \cdot\left(u_{1}^{\varepsilon}-u_{2}^{\varepsilon}\right) d \Gamma=0 .
$$

By the monotonicity of the function $\sigma^{\varepsilon}(x, u)$ (property $a_{1}$ ), we get

$$
u_{1}^{\varepsilon}=u_{2}^{\varepsilon} \text { almost everywhere in } \Omega^{\varepsilon} .
$$

This proves the uniqueness of the generalized solution of boundary-value problem (1). Theorem 1 is proved.

\section{Auxiliary Statements}

The proof of the main theorem is based on the variational methods. The main idea is to construct suitable approximations of the generalized solution $u^{\varepsilon}(x)$ of problem (1). These approximations are constructed by using the minimizers of the functionals (8) and (12). In order to implement this method, as a preliminary, we prove several statements characterizing the minimizers themselves, as well as the functions constructed with their help.

Lemma 2. Let conditions 1), 2) of Theorem 2 be satisfied. Then there exist the sequences $\left\{\varepsilon_{k}\right\}_{k=1}^{\infty},\left\{h_{k}^{\prime}\right\}_{k=1}^{\infty},\left\{h_{k}^{\prime \prime}=h_{k}^{\prime}+\left(h_{k}^{\prime}\right)^{1+\tau / 2}\right\}_{k=1}^{\infty}$, such that $\varepsilon_{k}, h_{k}^{\prime}, h_{k}^{\prime \prime} \rightarrow$ 0 as $k \rightarrow \infty$ and, for almost all $x \in \Omega$, the following equalities hold 
1) $\lim _{k \rightarrow \infty} \frac{a_{i j}\left(x, \varepsilon_{k}, h_{k}^{\prime}\right)}{\left(h_{k}^{\prime}\right)^{n}}=\lim _{k \rightarrow \infty} \frac{a_{i j}\left(x, \varepsilon_{k}, h_{k}^{\prime \prime}\right)}{\left(h_{k}^{\prime \prime}\right)^{n}}=a_{i j}(x), i, j=1, \ldots, n$;

2) $\lim _{k \rightarrow \infty} \frac{c\left(x, s, \varepsilon_{k}, h_{k}^{\prime}\right)}{\left(h_{k}^{\prime}\right)^{n}}=\lim _{k \rightarrow \infty} \frac{c\left(x, s, \varepsilon_{k}, h_{k}^{\prime \prime}\right)}{\left(h_{k}^{\prime \prime}\right)^{n}}=c(x, s), \forall s \in R^{1}$;

3) for every $\mu>0$, there exists a closed set $\Omega_{\mu} \subset \Omega$ such that mes $\left(\Omega \backslash \Omega_{\mu}\right)<\mu$ and assertions 1), 2) above are satisfied uniformly with respect to $x \in \Omega_{\mu}$.

Proof. From conditions 1), 2) of Theorem 2, it follows that there exists a monotone function $\widehat{\varepsilon}(h)$ such that $\widehat{\varepsilon}(h) \rightarrow 0$ as $h \rightarrow 0$ and, for all $\varepsilon_{h}<\widehat{\varepsilon}(h)$,

$\lim _{h \rightarrow 0} \int_{\Omega}\left|\frac{a_{i j}\left(x, \varepsilon_{h}, h\right)}{h^{n}}-a_{i j}(x)\right| d x=0=\lim _{h \rightarrow 0} \int_{\Omega}\left|\frac{c\left(x, s ; \varepsilon_{h}, h\right)}{h^{n}}-c(x, s)\right| d x$.

Hence, taking into account that mean convergence implies convergence almost everywhere on the subsequence $[11$, p. $5, \S 4]$, we conclude that there exists a sequence $\left\{h_{l}^{\prime}\right\}_{l=1}^{\infty}$ such that $h_{l}^{\prime} \rightarrow 0$ as $l \rightarrow \infty$ and, for almost all $x \in \Omega$,

$$
\begin{array}{ll}
\lim _{l \rightarrow \infty} \frac{a_{i j}\left(x, \varepsilon_{l}, h_{l}^{\prime}\right)}{\left(h_{l}^{\prime}\right)^{n}}=a_{i j}(x), & i, j=1, \ldots, n ; \\
\lim _{l \rightarrow \infty} \frac{c\left(x, s, \varepsilon_{l}, h_{l}^{\prime}\right)}{\left(h_{l}^{\prime}\right)^{n}}=c(x, s), & \forall s \in R^{1},
\end{array}
$$

where $\varepsilon_{l}=\widehat{\varepsilon}\left(h_{l}^{\prime}\right)$.

Setting in $(20) h=h_{l}^{\prime \prime}=h_{l}^{\prime}+\left(h_{l}^{\prime}\right)^{1+\tau / 2}(\tau>0)$, we have

$$
\lim _{l \rightarrow \infty} \int_{\Omega}\left|\frac{a_{i j}\left(x, \varepsilon_{l}, h_{l}^{\prime \prime}\right)}{\left(h_{l}^{\prime \prime}\right)^{n}}-a_{i j}(x)\right| d x=0=\lim _{l \rightarrow \infty} \int_{\Omega}\left|\frac{c\left(x, s ; \varepsilon_{l}, h_{l}^{\prime \prime}\right)}{\left(h_{l}^{\prime \prime}\right)^{n}}-c(x, s)\right| d x .
$$

Then from the sequence $\left\{h_{l}^{\prime}\right\}$ we can choose a subsequence $\left\{h_{k}^{\prime}\right\} \subset\left\{h_{l}^{\prime}\right\}$ and, consequently, the subsequences $\left\{h_{k}^{\prime \prime}\right\} \subset\left\{h_{l}^{\prime \prime}\right\}$ and $\left\{\varepsilon_{k}=\widehat{\varepsilon}\left(h_{k}^{\prime}\right)\right\}$, for which the assertions 1), 2) of the lemma hold.

Applying Egorov's theorem [11], we obtain the third assertion of the lemma. The lemma is proved.

Further, since we will consider the convergence on the sequences $\left\{\varepsilon_{k}\right\}_{k=1}^{\infty}$, $\left\{h_{k}^{\prime}\right\}_{k=1}^{\infty}$ and $\left\{h_{k}^{\prime \prime}\right\}_{k=1}^{\infty}$ constructed in Lemma 2, for the for the sake of brevity, we use the following notation:

$$
\begin{aligned}
& \Pi_{k}^{z}:=K_{h_{k}^{\prime \prime}}^{z} \backslash K_{h_{k}^{\prime}}^{z}, \quad K_{k}^{z}:=K_{h_{k}^{\prime \prime}}^{z}, \quad \Omega^{k}:=\Omega^{\varepsilon_{k}}, \quad \partial F^{k}:=\partial F^{\varepsilon_{k}}, \\
& g^{k}(x, u):=g^{\varepsilon_{k}}(x, u), \quad \sigma^{k}(x, u):=\sigma^{\varepsilon_{k}}(x, u), \quad f^{k}(x):=f^{\varepsilon_{k}}(x) .
\end{aligned}
$$


Lemma 3. Let condition 1) of Theorem 2 be satisfied, $\left\{\varepsilon_{k}\right\}_{k=1}^{\infty},\left\{h_{k}^{\prime}\right\}_{k=1}^{\infty}$, $\left\{h_{k}^{\prime \prime}=h_{k}^{\prime}+\left(h_{k}^{\prime}\right)^{1+\tau / 2}\right\}_{k=1}^{\infty}$ and $\Omega_{\mu}$ be the sequences and the set, respectively, constructed in Lemma 2, the function $v_{k}^{z}$ minimizes functional (8) in the domain $K_{k}^{z} \cap \Omega^{k}$ for $\ell \in R^{n}$.

Then, for every $\mu>0$,

$$
\begin{aligned}
& \lim _{k \rightarrow \infty} \frac{1}{\left(h_{k}^{\prime \prime}\right)^{n}} \int_{\Pi_{k}^{z} \cap \Omega^{k}}\left|\nabla v_{k}^{z}(x)\right|^{2} d x=0, \\
& \lim _{k \rightarrow \infty} \frac{1}{\left(h_{k}^{\prime \prime}\right)^{n+2+\tau}} \int_{\Pi_{k}^{z} \cap \Omega^{k}}\left|v_{k}^{z}(x)-(x-z, \ell)\right|^{2} d x=0, \\
& \lim _{k \rightarrow \infty} \sum_{i, j=1}^{n} \frac{1}{\left(h_{k}^{\prime \prime}\right)^{n}} \int_{K_{k}^{z} \cap \Omega^{k}}\left(\nabla v_{k i}^{z}, \nabla v_{k j}^{z}\right) \ell_{i} \ell_{j} d x \leqslant \sum_{i, j=1}^{n} a_{i j}(z) \ell_{i} \ell_{j}
\end{aligned}
$$

uniformly with respect to $z \in \Omega_{\mu}$.

Proof. Let $v_{k i}^{z}(x)$ be a minimizer of functional (8) in the domain $K_{k}^{z} \cap \Omega^{k}$ as $\ell \in R^{n}$ ( $e^{i}$ is a basic vector of the axis $\left.x_{i}(i=1, \ldots, n)\right)$. Using the definition of functional (8), its representation (10) and the expression for the coefficients $a_{i j}(z, \varepsilon, h)(11)$, we obtain

$$
\begin{aligned}
& \frac{1}{\left(h_{k}^{\prime \prime}\right)^{n}} \int_{\Pi_{k}^{z} \cap \Omega^{k}}\left(\left|\nabla v_{k i}^{z}\right|^{2}+\left(h_{k}^{\prime \prime}\right)^{-2-\tau}\left|v_{k i}^{z}-\left(x_{i}-z_{i}\right)\right|^{2}\right) d x \\
& =\frac{a_{i i}\left(z, \varepsilon_{k}, h_{k}^{\prime \prime}\right)}{\left(h_{k}^{\prime \prime}\right)^{n}}-\frac{1}{\left(h_{k}^{\prime \prime}\right)^{n}} \int_{K_{h_{k}^{\prime}}^{z} \cap \Omega^{k}}\left(\left|\nabla v_{k i}^{z}\right|^{2}+\left(h_{k}^{\prime \prime}\right)^{-2-\tau}\left|v_{k i}^{z}-\left(x_{i}-z_{i}\right)\right|^{2}\right) d x \\
& \quad \leqslant \frac{a_{i i}\left(z, \varepsilon_{k}, h_{k}^{\prime \prime}\right)}{\left(h_{k}^{\prime \prime}\right)^{n}}-\frac{\left(h_{k}^{\prime}\right)^{n}}{\left(h_{k}^{\prime \prime}\right)^{n}} \cdot \frac{a_{i i}\left(z, \varepsilon_{k}, h_{k}^{\prime}\right)}{\left(h_{k}^{\prime}\right)^{n}} .
\end{aligned}
$$

Furthermore,

$$
\frac{1}{\left(h_{k}^{\prime \prime}\right)^{n}} \int_{K_{k}^{z} \cap \Omega^{k}}\left(\nabla v_{k i}^{z}, \nabla v_{k j}^{z}\right) \ell_{i} \ell_{j} d x \leqslant \frac{a_{i j}\left(z, \varepsilon_{k}, h_{k}^{\prime \prime}\right)}{\left(h_{k}^{\prime \prime}\right)^{n}} \ell_{i} \ell_{j} .
$$

This, Lemma 2 implies that

$$
\begin{aligned}
& \lim _{k \rightarrow \infty} \frac{1}{\left(h_{k}^{\prime \prime}\right)^{n}} \int_{\Pi_{k}^{z} \cap \Omega^{k}}\left|\nabla v_{k i}^{z}\right|^{2} d x=0, \\
& \lim _{k \rightarrow \infty} \frac{1}{\left(h_{k}^{\prime \prime}\right)^{n+2+\tau}} \int_{\Pi_{k}^{z} \cap \Omega^{k}}\left|v_{k i}^{z}-\left(x_{i}-z_{i}\right)\right|^{2} d x=0, \\
& \lim _{k \rightarrow \infty} \frac{1}{\left(h_{k}^{\prime \prime}\right)^{n}} \int_{K_{k}^{z} \cap \Omega^{k}}\left(\nabla v_{k i}^{z}, \nabla v_{k j}^{z}\right) \ell_{i} \ell_{j} d x \leqslant a_{i j}(z) \ell_{i} \ell_{j}
\end{aligned}
$$

uniformly in $z \in \Omega_{\mu}$. Since $v_{k}^{z}=\sum_{i=1}^{n} v_{k i}^{z} \cdot \ell_{i}$ and $\ell=\sum_{i=1}^{n} \ell_{i} e^{i}$, the assertions of the lemma are valid. The lemma is proved. 
Lemma 4. Let condition 2) of Theorem 2 be satisfied, $\left\{\varepsilon_{k}\right\}_{k=1}^{\infty},\left\{h_{k}^{\prime}\right\}_{k=1}^{\infty}$, $\left\{h_{k}^{\prime \prime}=h_{k}^{\prime}+\left(h_{k}^{\prime}\right)^{1+\tau / 2}\right\}_{k=1}^{\infty}$ and $\Omega_{\mu}$ be the sequences and the set, respectively, constructed in Lemma 2.

Then

1) for every $s=\widehat{s}$, there exists a unique minimizer $w_{k}^{z}$ of functional (12) in the domain $K_{k}^{z} \cap \Omega^{k}$, and it satisfies the estimate

$$
\left|w_{k}^{z}(x)\right| \leqslant|\widehat{s}|
$$

2) for every $\mu>0$,

$$
\begin{aligned}
& \lim _{k \rightarrow \infty} \frac{1}{\left(h_{k}^{\prime \prime}\right)^{n}} \int_{\Pi_{k}^{z} \cap \Omega^{k}}\left|\nabla w_{k}^{z}(x)\right|^{2} d x=0, \\
& \lim _{k \rightarrow \infty} \frac{1}{\left(h_{k}^{\prime \prime}\right)^{n+2+\tau}} \int_{\Pi_{k}^{z} \cap \Omega^{k}}\left|w_{k}^{z}(x)-\widehat{s}\right|^{2} d x=0, \\
& \lim _{k \rightarrow \infty} \frac{1}{\left(h_{k}^{\prime \prime}\right)^{n}} \int_{\Pi_{k}^{z} \cap \partial F^{k}}, g^{\varepsilon}\left(x, w_{k}^{z}\right) d \Gamma=0, \\
& \lim _{k \rightarrow \infty} \frac{1}{\left(h_{k}^{\prime \prime}\right)^{n+2+\tau}} \int_{K_{k}^{z} \cap \Omega^{k}}\left|w_{k}^{z}-\widehat{s}\right|^{2} d x \leqslant C, \\
& \lim _{k \rightarrow \infty} \frac{1}{\left(h_{k}^{\prime \prime}\right)^{n}}\left\{\int_{K_{k}^{z} \cap \Omega^{k}}\left|\nabla w_{k}^{z}\right|^{2} d x+\int_{K_{k}^{z} \cap \partial F^{k}} g^{k}\left(x, w_{k}^{z}\right) d \Gamma\right\} \leqslant c(z, \widehat{s})
\end{aligned}
$$

uniformly with respect to $z \in \Omega_{\mu}$;

3) for every $\delta>0$, a measure of the set $B_{k}^{z \delta}=\left\{x \in K_{k}^{z} \cap \Omega^{k}:\left|w_{k}^{z}-\widehat{s}\right| \geqslant \delta\right\}$ satisfies the estimate

$$
\operatorname{mes}\left\{B_{k}^{z \delta}\right\}<\frac{C\left(h_{k}^{\prime \prime}\right)^{n+2+\tau}}{\delta^{2}} .
$$

Proof. The existence and the uniqueness of $w_{k}^{z} \in H^{1}\left(K_{h}^{z} \cap \Omega^{k}\right)$ can be proved by the standard variational methods (see, for example, the proof of Theorem 1). Let us prove the estimate for the function $w_{k}^{z}$. For simplicity, we set $\widehat{s}>0$ and assume that estimate 1 ) is not true. Then in the domain $K_{k}^{z} \cap \Omega^{k}$ there exists a set $E$ on which $w_{k}^{z}>\widehat{s}$. We construct a cut-off function $\widehat{w}_{k}^{z}$ such that $\widehat{w}_{k}^{z}=\widehat{s}$ for $x \in E$ and $\widehat{w}_{k}^{z}=w_{k}^{z}$ for $x \in\left(K_{k}^{z} \cap \Omega^{k}\right) \backslash E$. The function $\widehat{w}_{k}^{z}$ provides a smaller value for functional (12) than the function $w_{k}^{z}$. This contradicts our assumption that $w_{k}^{z}$ is a minimizer of this functional.

Next we prove assertion 2) of the lemma. By (12), we obtain

$$
\frac{1}{\left(h_{k}^{\prime \prime}\right)^{n}}\left[\int_{\Pi_{k}^{z} \cap \Omega^{k}}\left(\left|\nabla w_{k}^{z}\right|^{2}+\left(h_{k}^{\prime \prime}\right)^{-2-\tau}\left|w_{k}^{z}-\widehat{s}\right|^{2}\right) d x+\int_{\Pi_{k}^{z} \cap \partial F^{k}} g^{k}\left(x, w_{k}^{z}\right) d \Gamma\right]
$$




$$
\begin{gathered}
=\frac{c\left(z, \widehat{s} ; \varepsilon_{k}, h_{k}^{\prime \prime}\right)}{\left(h_{k}^{\prime \prime}\right)^{n}}-\frac{1}{\left(h_{k}^{\prime \prime}\right)^{n}}\left[\int_{K_{h_{k}^{\prime}}^{z} \cap \Omega^{k}}\left(\left|\nabla w_{k}^{z}\right|^{2}+\left(h_{k}^{\prime \prime}\right)^{-2-\tau}\left|w_{k}^{z}-\widehat{s}\right|^{2}\right) d x\right. \\
\left.\quad+\int_{K_{h_{k}^{\prime}} \cap \partial F^{k}} g^{k}\left(x, w_{k}^{z}\right) \Gamma\right] \leqslant \frac{c\left(z, \widehat{s} ; \varepsilon_{k}, h_{k}^{\prime \prime}\right)}{\left(h_{k}^{\prime \prime}\right)^{n}}-\frac{\left(h_{k}^{\prime}\right)^{n}}{\left(h_{k}^{\prime \prime}\right)^{n}} \cdot \frac{c\left(z, \widehat{s} ; \varepsilon_{k}, h_{k}^{\prime}\right)}{\left(h_{k}^{\prime}\right)^{n}} .
\end{gathered}
$$

We pass to the limit as $k \rightarrow \infty$. Since all the integrands are positive and assertion 2) of Lemma 2 is true, then the limit equalities of 2) are satisfied uniformly with respect to $z \in \Omega_{\mu}$.

In addition, using (12) and assertion 2) of Lemma 2, we have as $k \rightarrow \infty$,

$$
\begin{array}{r}
\frac{1}{\left(h_{k}^{\prime \prime}\right)^{n}}\left[\int_{K_{k}^{z} \cap \Omega^{k}}\left(\left|\nabla w_{k}^{z}\right|^{2}+\left(h_{k}^{\prime \prime}\right)^{-2-\tau}\left|w_{k}^{z}-\widehat{s}\right|^{2}\right) d x+\int_{K_{k}^{z} \cap \partial F^{k}} g^{k}\left(x, w_{k}^{z}\right) d \Gamma\right] \\
=c(z, \widehat{s})+o(1) \leqslant C .
\end{array}
$$

Therefore,

$$
\frac{1}{\left(h_{k}^{\prime \prime}\right)^{n}}\left[\int_{K_{k}^{z} \cap \Omega^{k}}\left|\nabla w_{k}^{z}\right|^{2} d x+\int_{K_{k}^{z} \cap \partial F^{k}} g^{k}\left(x, w_{k}^{z}\right) d \Gamma\right] \leqslant c(z, \widehat{s})+o(1), k \rightarrow \infty
$$

and

$$
\int_{K_{k}^{z} \cap \Omega^{k}}\left|w_{k}^{z}-\widehat{s}\right|^{2} d x \leqslant C\left(h_{k}^{\prime \prime}\right)^{n+2+\tau} .
$$

This establishes the limit inequalities of 2) and the third assertion of the lemma. The lemma is proved.

Lemma 5. Let condition 2) of Theorem 2 be satisfied, $\left\{\varepsilon_{k}\right\}_{k=1}^{\infty},\left\{h_{k}^{\prime}\right\}_{k=1}^{\infty}$, $\left\{h_{k}^{\prime \prime}=h_{k}^{\prime}+\left(h_{k}^{\prime}\right)^{1+\tau / 2}\right\}_{k=1}^{\infty}$ and $\Omega_{\mu}$ be the sequences and the set, respectively, constructed in Lemma 2. Let the function $w_{k}^{z}$ minimizes functional (12) in the domain $K_{k}^{z} \cap \Omega^{k}$ for $s=\widehat{s}$.

Then the set

$$
B_{k}^{z}=\left\{x \in K_{k}^{z} \cap \Omega^{k}:\left|w_{k}^{z}-\widehat{s}\right| \geqslant\left(h_{k}^{\prime \prime}\right)^{1+\tau / 3}\right\}
$$

and the function

$$
\widehat{w}_{k}^{z}=\left\{\begin{array}{lc}
w_{k}^{z}, & x \in B_{k}^{z}, \\
\widehat{s}-\left(h_{k}^{\prime \prime}\right)^{1+\tau / 3}, & \widehat{s} \geqslant 0, x \in\left(K_{k}^{z} \cap \Omega^{k}\right) \backslash B_{k}^{z}, \\
\widehat{s}+\left(h_{k}^{\prime \prime}\right)^{1+\tau / 3}, & \widehat{s} \leqslant 0, x \in\left(K_{k}^{z} \cap \Omega^{k}\right) \backslash B_{k}^{z},
\end{array}\right.
$$

satisfy the properties: 
1) a measure of $B_{k}^{z}$,

$$
\operatorname{mes}\left\{B_{k}^{z}\right\}<C\left(h_{k}^{\prime \prime}\right)^{n+\tau / 3}
$$

2) for every $\mu>0$,

$$
\begin{aligned}
& \lim _{k \rightarrow \infty} \frac{1}{\left(h_{k}^{\prime \prime}\right)^{n}} \int_{\Pi_{k}^{z} \cap \Omega^{k}}\left|\nabla \widehat{w}_{k}^{z}(x)\right|^{2} d x=0, \\
& \lim _{k \rightarrow \infty} \frac{1}{\left(h_{k}^{\prime \prime}\right)^{n+2+\tau}} \int_{\Pi_{k}^{z} \cap \Omega^{k}}\left|\widehat{w}_{k}^{z}(x)-\widehat{s}\right|^{2} d x=0, \\
& \lim _{k \rightarrow \infty} \frac{1}{\left(h_{k}^{\prime \prime}\right)^{n}} \int_{\Pi_{k}^{z} \cap \partial F^{k}} g^{k}\left(x, \widehat{w}_{k}^{z}\right) d \Gamma=0, \\
& \lim _{k \rightarrow \infty} \frac{1}{\left(h_{k}^{\prime \prime}\right)^{n+2+\tau}} \int_{K_{k}^{z} \cap \Omega^{k}}\left|\widehat{w}_{k}^{z}-\widehat{s}\right|^{2} d x \leqslant C, \\
& \lim _{k \rightarrow \infty} \frac{1}{\left(h_{k}^{\prime \prime}\right)^{n}}\left\{\int_{K_{k}^{z} \cap \Omega^{k}}\left|\nabla \widehat{w}_{k}^{z}\right|^{2} d x+\int_{K_{k}^{z} \cap \partial F^{k}} g^{k}\left(x, \widehat{w}_{k}^{z}\right) d \Gamma\right\} \leqslant c(z, \widehat{s})
\end{aligned}
$$

uniformly with respect to $z \in \Omega_{\mu}$.

Proof. The first assertion of the lemma follows from conclusion 3) of Lemma 4.

We proceed to prove the second assertion of the lemma. Without loss of generality, with regard for smallness of $h_{k}^{\prime \prime}$, we assume that $\widehat{s} \geqslant\left(h_{k}^{\prime \prime}\right)^{1+\tau / 3}>0$. By (13), (22) and the properties $a_{1}, a_{2}$ of the function $\sigma^{k}(x, u)$, the functions $\widehat{w}_{k}^{z}$, $g^{k}\left(x, \widehat{w}_{k}^{z}\right)$ satisfy the estimates

$$
\begin{aligned}
\left|\nabla \widehat{w}_{k}^{z}(x)\right|^{2} & \leqslant\left|\nabla w_{k}^{z}(x)\right|^{2} \\
\left|\widehat{w}_{k}^{z}(x)-\widehat{s}\right|^{2} & \leqslant\left|w_{k}^{z}(x)-\widehat{s}\right|^{2}+\left(h_{k}^{\prime \prime}\right)^{2+2 \tau / 3} \\
g^{k}\left(x, \widehat{w}_{k}^{z}\right) & =g^{k}\left(x, w_{k}^{z}\right)+O\left(\left(h_{k}^{\prime \prime}\right)^{1+\tau / 3}\right)
\end{aligned}
$$

for every $x \in K_{k}^{z} \cap \Omega^{k}$.

From these estimates and the conclusions of part 2) of Lemma 4, it follows that the second assertion of the lemma is valid for $\widehat{s}>0$. Similarly, we consider the case $\widehat{s}<0$. The lemma is proved.

Lemma 6. Let the domain $\Omega_{\mu}$ be a subdomain of $\Omega$. Then we can construct a covering of the domain $\Omega$ with disjoint cubes $K_{h}^{\alpha}$ of the size $h$ centered at $x^{\alpha}$, $\alpha=1, \ldots, N_{k} \geqslant \frac{\operatorname{mes}\{\Omega\}}{h^{n}}$, such that the number of cubes with centers not belonging to the domain $\Omega_{\mu}$ satisfy the inequality

$$
N_{k}^{\prime} \leqslant \frac{\operatorname{mes}\left\{\Omega \backslash \Omega_{\mu}\right\}}{\operatorname{mes}\{\Omega\}} N_{k}
$$


Proof. Let us cover $\Omega$ with disjoint (having no common interior points) cubes $\widetilde{K}_{h}^{\alpha}=K\left(\widetilde{x}^{\alpha}, h\right)$ of the size $h$ centered at $\widetilde{x}^{\alpha}$. Let $\chi^{\alpha}$ be a characteristic function of the domain $\left(\Omega \backslash \Omega_{\mu}\right) \cap \widetilde{K}_{h}^{\alpha}$. We have

$$
\operatorname{mes}\left\{\Omega \backslash \Omega_{\mu}\right\}=\sum_{\alpha} \int_{\widetilde{K}_{h}^{\alpha}} \chi^{\alpha}(x) d x .
$$

With the help of shift, we combine all the cubes $\widetilde{K}_{h}^{\alpha}$ in one $K_{h}^{0}$ centered at 0 . Thus, we get

$$
\sum_{\alpha} \int_{\widetilde{K}_{h}^{\alpha}} \chi^{\alpha}(x) d x=\sum_{\alpha} \int_{K_{h}^{0}} \chi^{\alpha}\left(x-\widetilde{x}^{\alpha}\right) d x=\int_{K_{h}^{0}} \sum_{\alpha} \chi^{\alpha}\left(x-\widetilde{x}^{\alpha}\right) d x .
$$

We denote

$$
\chi(x)=\sum_{\alpha} \chi^{\alpha}\left(x-\widetilde{x}^{\alpha}\right) .
$$

It is clear that $\chi(x)$ is an integer-valued function in $K_{h}^{0}$, which determines the multiplicity of coverage of the point $x \in K_{h}^{0}$ by the image of the set $\Omega \backslash \Omega_{\mu}$. We choose a point $\widehat{x} \in K_{h}^{0}$ with the smallest coverage multiplicity $N_{k}^{\prime}$. Hence,

$$
\int_{K_{h}^{0}} \chi(x) d x \geqslant N_{k}^{\prime} h^{n} .
$$

We take the points $x^{\alpha}$ as unknown centers of the cubes $K_{h}^{\alpha}$, which are the images of the point $\widehat{x}$ under reverse shift. The number of the centers of the cubes in the set $\Omega \backslash \Omega_{\mu}$ is equal to $N_{k}^{\prime}$. Suppose that inequality (23) does not hold, then by (24)-(27), we get

$$
\operatorname{mes}\left\{\Omega \backslash \Omega_{\mu}\right\} \geqslant N_{k}^{\prime} h^{n}>\frac{\operatorname{mes}\left\{\Omega \backslash \Omega_{\mu}\right\}}{\operatorname{mes}\{\Omega\}} N_{k} h^{n} \geqslant \operatorname{mes}\left\{\Omega \backslash \Omega_{\mu}\right\} .
$$

We have obtained a contradiction

$$
\operatorname{mes}\left\{\Omega \backslash \Omega_{\mu}\right\}>\operatorname{mes}\left\{\Omega \backslash \Omega_{\mu}\right\} .
$$

Thus inequality (23) is true. The lemma is proved

Lemma 7. Let the sets $B_{k} \subset \Omega^{k} \subset \Omega$ be given and

$$
\lim _{k \rightarrow \infty} \operatorname{mes}\left\{B_{k}\right\}=0
$$

If condition 1) of Theorem 2 is fulfilled, then there exists a set $\widehat{B}_{k} \subset \Omega$ and the functions $\widehat{v}_{k i} \in H^{1}(\Omega)(i=\overline{1, n})$ satisfying the following conditions: 
1) $B_{k} \subset \widehat{B}_{k}, \lim _{k \rightarrow \infty} \operatorname{mes}\left\{\widehat{B}_{k}\right\}=0$;

2) $\lim _{k \rightarrow \infty} \max _{x \in \Omega}\left|\widehat{v}_{k i}(x)-x_{i}\right|=0$;

3) $\lim _{k \rightarrow \infty} \int_{\widehat{B}_{k}}\left|\nabla \widehat{v}_{k i}\right|^{2} d x=0$;

4) for any vector function $\ell(x)=\left(\ell_{1}(x), \ldots, \ell_{n}(x)\right) \in(C(\bar{\Omega}))^{n}$,

$$
\lim _{k \rightarrow \infty} \int_{\Omega^{k}} \sum_{i, j=1}^{n}\left(\nabla \widehat{v}_{k i}, \nabla \widehat{v}_{k j}\right) \ell_{i}(x) \ell_{j}(x) d x \leqslant \int_{\Omega} \sum_{i, j=1}^{n} a_{i j}(x) \ell_{i}(x) \ell_{j}(x) d x .
$$

Proof. Let $\left\{\varepsilon_{k}\right\}_{k=1}^{\infty},\left\{h_{k}^{\prime}\right\}_{k=1}^{\infty},\left\{h_{k}^{\prime \prime}=h_{k}^{\prime}+\left(h_{k}^{\prime}\right)^{1+\tau / 2}\right\}_{k=1}^{\infty}$ be the sequences constructed in Lemma 2. Let $\left\{\mu_{k}\right\}_{k}$ be the sequence of numbers and $\mu_{k} \rightarrow 0$ as $k \rightarrow \infty$. Due to assertion 3) of Lemma 2, we can construct a sequence of sets $\Omega_{\mu_{1}} \subset \Omega_{\mu_{2}} \subset \cdots \subset \Omega_{\mu_{k}} \subset \cdots \subset \Omega$ such that $\operatorname{mes}\left\{\Omega \backslash \Omega_{\mu_{k}}\right\}<\mu_{k}$.

Let us cover the domain $\Omega$ by disjoint cubes $K_{h_{k}}^{\alpha}\left(\Omega \in \cup_{\alpha} K_{h_{k}}^{\alpha}\right)$ of the size $h_{k}=\frac{h_{k}^{\prime \prime}+h_{k}^{\prime}}{2}$ centered at the points $x^{\alpha}$ as in Lemma 6 . Besides the cubes $K_{h_{k}}^{\alpha}$, we also consider the cubes $K_{h_{k}^{\prime}}^{\alpha}$ and $K_{h_{k}^{\prime \prime}}^{\alpha}$. With this covering, we associate a partition of the unity $\left\{\varphi_{k}^{\alpha}(x)\right\}_{\alpha}$ of twice continuously differentiable functions satisfying the following conditions:

$$
\begin{aligned}
& \varphi_{k}^{\alpha}(x)=0, \quad \text { if } x \notin K_{h_{k}^{\prime \prime}}^{\alpha} ; \quad \varphi_{k}^{\alpha}(x)=1, \quad \text { if } x \in K_{h_{k}^{\prime}}^{\alpha} ; \\
& \forall x \in \Omega: 0 \leqslant \varphi_{k}^{\alpha}(x) \leqslant 1, \quad \sum_{\alpha} \varphi_{k}^{\alpha}(x)=1, \quad\left|D \varphi_{k}^{\alpha}(x)\right|<C\left(h_{k}^{\prime}\right)^{-1-\tau / 2} .
\end{aligned}
$$

In each cube $K_{k}^{\alpha}:=K_{h_{k}^{\prime \prime}}^{\alpha}$, we construct the set $B_{k}^{\alpha}$ by Lemma 5 and the set $B_{k}=\cup_{\alpha} B_{k}^{\alpha}$, whose measure, by virtue of assertion 1) of Lemma 5 , satisfies the inequality

$$
\operatorname{mes}\left\{B_{k}\right\} \leqslant \sum_{\alpha} \operatorname{mes}\left\{B_{k}^{\alpha}\right\}<C\left(h_{k}^{\prime \prime}\right)^{\tau / 3} .
$$

The set $B_{k}$ satisfies the condition of the lemma.

Let $v_{k i}^{\alpha}(i=\overline{1, n})$ be a minimizer of (8) in the cube $K_{k}^{\alpha}$ with $x^{\alpha} \in \Omega_{\mu_{k}}$ for $\ell=$ $e^{i}$. Then, by the maximum principle,

$$
\max _{x \in K_{k}^{\alpha} \cap \Omega^{k}}\left|v_{k i}^{\alpha}(x)\right| \leqslant \frac{1}{2} h_{k}^{\prime \prime},
$$

and the functions $v_{k i}^{\alpha}(i=\overline{1, n})$ satisfy the assertions of Lemma 3 .

In $\Omega^{k}$, we consider the functions

$$
v_{k i}(x)=x_{i}+\sum_{\alpha}\left[v_{k i}^{\alpha}(x)-\left(x_{i}-x_{i}^{\alpha}\right)\right] \varphi_{k}^{\alpha}(x) \quad(i=\overline{1, n}),
$$


where $\left\{\varphi_{k}^{\alpha}(x)\right\}_{\alpha}$ is a partition of unity (28). Notice that $v_{k i}^{\alpha}(x)=x_{i}-x_{i}^{\alpha}$ if $x^{\alpha} \in$ $\Omega \backslash \Omega_{\mu_{k}}$ or the cube $K_{k}^{\alpha}$ does not lie entirely in $\Omega$.

It is clear that

$$
\frac{\partial v_{k i}(x)}{\partial x_{j}}=\sum_{\alpha} \frac{\partial v_{k i}^{\alpha}(x)}{\partial x_{j}} \varphi_{k}^{\alpha}(x)+\sum_{\alpha}\left[v_{k i}^{\alpha}(x)-\left(x_{i}-x_{i}^{\alpha}\right)\right] \frac{\partial \varphi_{k}^{\alpha}(x)}{\partial x_{j}} .
$$

Let $\ell(x)=\left\{\ell_{1}(x), \ell_{2}(x), \ldots, \ell_{n}(x)\right\}$ be an arbitrary vector function continuous in $\bar{\Omega}$. By Lemma 3 and the properties of the function $\varphi_{k}^{\alpha}(x)$, we obtain

$$
\int_{\Omega^{k}} \sum_{i, j=1}^{n}\left(\nabla v_{k i}, \nabla v_{k j}\right) \ell_{i}(x) \ell_{k}(x) d x \leqslant \int_{\Omega} \sum_{i, j=1}^{n} a_{i j}(x) \ell_{i}(x) \ell_{j}(x)+o(1), k \rightarrow \infty .
$$

Furthermore, by (29) and (30), we have

$$
\max _{x \in \Omega^{k}}\left|v_{k i}-x_{i}\right| \leqslant \frac{1}{2} h_{k}^{\prime \prime}
$$

Set $u_{k i}=v_{k i}-x_{i}$. Then from (31) and (32), we obtain the estimates

$$
\max _{x \in \Omega^{k}}\left|u_{k i}\right| \leqslant \frac{1}{2} h_{k}^{\prime \prime} \text { and }\left\|u_{k i}\right\|_{H^{1}\left(\Omega^{k}\right)} \leqslant C
$$

where $C$ is independent of $k$.

Since the domains $\Omega^{k}$ satisfy the extension condition (6), there exists a function $\bar{u}_{k i}(x) \in H^{1}(\Omega)$ equal to $u_{k i}(x)$ for $x \in \Omega^{k}$ which satisfies inequalities (33) in $\Omega$. We extend $\bar{u}_{k i}(x)$, maintaining these inequalities, on the parallelepiped $\Pi \supset$ $\Omega$ and then approximate a twice continuously differentiable in $\Pi$ function $\widetilde{u}_{k i}(x)$ such that

$$
\left\|\widetilde{u}_{k i}-\bar{u}_{k i}\right\|_{H^{1}(\Pi)} \leqslant \varepsilon_{k}, \quad \max _{x \in \Pi}\left|\widetilde{u}_{k i}\right| \leqslant C_{1} h_{k}^{\prime \prime}, \quad\left\|\widetilde{u}_{k i}\right\|_{H^{1}(\Pi)} \leqslant C_{2},
$$

where $C_{1}, C_{2}$ are independent of $k$.

Now we apply Lemma $1.3\left[12\right.$, Chap. 3] to the function $\widetilde{u}_{k i}(x)$ and the set $B_{k}$. By the lemma, there exists the function $\widehat{u}_{k i}(x) \in H^{1}(\Pi)$ and the set $\widehat{B}_{k} \subset \Pi$ satisfying the conditions:

$$
\begin{aligned}
& B_{k} \subset \widehat{B}_{k}, \quad \operatorname{mes}\left(\widehat{B}_{k}\right) \leqslant C_{1} A m^{2|\ln m|^{-1 / 3}}\left(m^{|\ln m|^{-2 / 3}}+|\ln m|^{-1 / 6}\right), \\
& \widehat{u}_{k i}(x)=\widetilde{u}_{k i}(x), \quad x \in \Pi \backslash \widehat{B}_{k}, \\
& \max _{x \in \Pi}\left|\widehat{u}_{k i}(x)\right| \leqslant C_{2} h_{k}^{\prime \prime}, \quad\left\|\widehat{u}_{k i}\right\|_{H^{1}\left(\widehat{B}_{k}\right)} \leqslant C_{3} A\left(m^{|\ln m|^{-2 / 3}}+|\ln m|^{-1 / 6}\right),
\end{aligned}
$$

where $m=\operatorname{mes}\left(B_{k}\right), A=\left\|\widetilde{u}_{k i}\right\|_{H^{1}(\Pi)}$ and the constants $C_{1}, C_{2}, C_{3}$ are independent of $k$. 
We set $\widehat{v}_{k i}=\widehat{u}_{k i}+x_{i}$ and consider the restrictions of the set $\widehat{B}_{k} \in \Pi$ and the function $\widehat{v}_{k i}(x) \in H^{1}(\Pi)$ on the domain $\Omega$ (preserving the original notation). We obtain the set and the function which by (34)-(36) satisfy assertions 1)-3) of the lemma. We now verify estimate 4) of the lemma. Set $\widetilde{v}_{k i}(x)=\widetilde{u}_{k i}(x)+$ $x_{i}$. According to (31), (34), and (36) taking into account the positivity of the integrand and estimate 3 ) of the lemma, the inequality

$$
\begin{aligned}
\int_{\Omega^{k}} \sum_{i, j=1}^{n}\left(\nabla \widehat{v}_{k i}, \nabla \widehat{v}_{k j}\right) \ell_{i}(x) \ell_{j}(x) d x & \\
& \leqslant \int_{\Omega} \sum_{i, j=1}^{n}\left(\nabla \widehat{v}_{k i}, \nabla \widehat{v}_{k j}\right) \ell_{i}(x) \ell_{j}(x) d x \\
& =\int_{\Omega \backslash \widehat{B}_{k}} \sum_{i, j=1}^{n}\left(\nabla \widetilde{v}_{k i}, \nabla \widetilde{v}_{k j}\right) \ell_{i}(x) \ell_{j}(x) d x \\
& =\int_{\Omega^{k}} \sum_{i, j=1}^{n}\left(\nabla v_{k i}, \nabla v_{k j}\right) \ell_{i}(x) \ell_{j}(x) d x+o(1) \\
& \leqslant \int_{\Omega} \sum_{i, j=1}^{n} a_{i j}(x) \ell_{i}(x) \ell_{j}(x)+o(1), \quad k \rightarrow \infty
\end{aligned}
$$

holds for all $\ell(x) \in(C(\bar{\Omega}))^{n}$. This proves estimate 4$)$. The lemma is proved.

\section{Proof of Main Theorem 2}

We briefly outline the scheme of the proof. It is similar to the scheme developed in [9], but it should be taken into account that the conditions of the uniform convergence does not hold in the entire region $\Omega$.

Earlier, in the proof of Theorem 1, we determined the energy functional $\Phi^{\varepsilon}[\cdot]$ (15) of the original problem (1). The generalized solution $u^{\varepsilon}(x)$ of problem (1) minimizes this functional in a class of functions $u^{\varepsilon}(x) \in H^{1}\left(\Omega^{\varepsilon}, \Omega\right)$.

We also determine an energy functional of homogenized problem (2),

$$
\Phi[u]=\int_{\Omega} \sum_{i, j=1}^{n} a_{i j} \frac{\partial u}{\partial x_{i}} \frac{\partial u}{\partial x_{j}} d x+\int_{\Omega} c(x, u) d x-2 \int_{\Omega} f u d x .
$$

The generalized solution of homogenized problem (2) minimizes $\Phi[u]$ in the class of functions $u(x) \in \stackrel{\circ}{H}^{1}(\Omega)$. 
In Subsection 5.1, we show that the generalized solutions $u^{\varepsilon}(x)$ of problem (1) can be extended on the set $F^{\varepsilon}$ such that the extended solutions $\widetilde{u}^{\varepsilon}(x)$ are uniformly bounded with respect to $\varepsilon$ in $\stackrel{\circ}{H}^{1}(\Omega)$. The resulting sequence $\left\{\widetilde{u}^{\varepsilon}(x)\right\}_{\varepsilon}$ is weakly compact in $\stackrel{\circ}{H}^{1}(\Omega)$. Hence, we can select the subsequence $\left\{\widetilde{u}^{\varepsilon_{m}}(x), m=1, \ldots, \infty\right\}$ that is weakly convergent in $\stackrel{\circ}{H}^{1}(\Omega)$ and, by the embedding theorem, strongly convergent in $L^{p}(\Omega)\left(p<\frac{2 n}{n-2}\right)$ to some function $u(x) \in$ $\stackrel{\circ}{H}^{1}(\Omega)$.

In Subsections 5.2-5.3, we prove that the function $u(x)$ is the minimizer of functional (37) and, consequently, the generalized solution of homogenized problem (2). This is done as follows. In Subsection 5.2, we define the special test function $w^{\varepsilon}(x)$ approximating the minimizer of functional (15). The function is constructed on an arbitrary function $w(x) \in C_{0}^{2}(\Omega)$. Since the solution $u^{\varepsilon}(x)$ of problem (1) minimizes functional (15) in $H^{1}\left(\Omega^{\varepsilon}, \partial \Omega\right)$, the inequality below holds,

$$
\Phi^{\varepsilon}\left[u^{\varepsilon}\right] \leqslant \Phi^{\varepsilon}\left[w^{\varepsilon}\right]
$$

Further we show that under the conditions of Theorem 2,

$$
\lim _{\varepsilon \rightarrow 0} \Phi^{\varepsilon}\left[w^{\varepsilon}\right]=\Phi[w]
$$

where $\Phi[\cdot]$ is defined by (37). By virtue (38), (39) and the density of $C_{0}^{2}(\Omega)$ in the space $\stackrel{\circ}{H}^{1}(\Omega)$, it follows that

$$
\varlimsup_{\varepsilon \rightarrow 0} \Phi^{\varepsilon}\left[u^{\varepsilon}\right] \leqslant \Phi[w], \forall w \in \stackrel{\circ}{H}^{1}(\Omega) .
$$

In Subsection 5.3, we show that if $u^{\varepsilon}(x)$ converges weakly in $\stackrel{\circ}{H}^{1}(\Omega)$ to a function $u(x)$ on some subsequence $\varepsilon=\varepsilon_{m} \rightarrow 0$, the reverse inequality holds,

$$
\varliminf_{\varepsilon=\bar{\varepsilon}_{m} \rightarrow 0} \Phi^{\varepsilon}\left[u^{\varepsilon}\right] \geqslant \Phi[u]
$$

From (40), (41), it follows that the limit function $u(x)$ satisfies the inequality $\Phi[u] \leqslant \Phi[w]$ for an arbitrary function $w(x) \in \stackrel{\circ}{H}^{1}(\Omega)$. Therefore, $u(x)$ minimizes the functional $\Phi[w]$ in the class $\stackrel{\circ}{H}^{1}(\Omega)$.

In Subsection 5.4, we prove that homogenized problem (2) has the unique generalized solution $u(x)$ and, therefore, the whole sequence of the extended solutions $\left\{\widetilde{u}^{\varepsilon}(x)\right\}_{\varepsilon}$ converges in $L^{p}(\Omega)$ to the function $u(x)$. Thus the sequence of solutions $\left\{u^{\varepsilon}(x)\right\}_{\varepsilon}$ of initial problem (1) converges in $L^{p}\left(\Omega^{\varepsilon}, \Omega\right)$ to the solution $u(x)$ of homogenized problem $(2)$. 
5.1. Compactness of the generalized solutions of problem (1). Since $\Phi^{\varepsilon}\left[u^{\varepsilon}\right] \leqslant \Phi^{\varepsilon}[0]=0$, the inequality

$$
0 \leqslant \int_{\Omega^{\varepsilon}}\left|\nabla u^{\varepsilon}\right|^{2} d x+\int_{\partial F^{\varepsilon}} g^{\varepsilon}\left(x, u^{\varepsilon}\right) d \Gamma \leqslant 2 \int_{\Omega^{\varepsilon}} f^{\varepsilon} u^{\varepsilon} d x
$$

is true. By virtue of the properties $a_{1}, a_{2}$ of the function $\sigma^{\varepsilon}(x, u)$, we have $g^{\varepsilon}\left(x, u^{\varepsilon}\right) \geqslant 0$.

By the Cauchy-Buniakovski inequality and nonnegativity of the function $g^{\varepsilon}\left(x, u^{\varepsilon}\right)$, we get

$$
\left\|\nabla u^{\varepsilon}\right\|_{L^{2}\left(\Omega^{\varepsilon}\right)}^{2} \leqslant 2\left\|f^{\varepsilon}\right\|_{L^{2}\left(\Omega^{\varepsilon}\right)}\left\|u^{\varepsilon}\right\|_{L^{2}\left(\Omega^{\varepsilon}\right)} .
$$

On account of the extension condition (5) and the Friedrichs inequality, there exists a function $\widetilde{u}^{\varepsilon}(x) \in \stackrel{\circ}{H}^{1}(\Omega)$ such that $\widetilde{u}^{\varepsilon}(x)=u^{\varepsilon}(x)$ for $x \in \Omega^{\varepsilon}$ and

$$
\left\|u^{\varepsilon}\right\|_{L^{2}(\Omega)} \leqslant\left\|\widetilde{u}^{\varepsilon}\right\|_{L^{2}(\Omega)} \leqslant C_{1}\left\|\nabla \widetilde{u}^{\varepsilon}\right\|_{L^{2}(\Omega)} \leqslant C_{2}\left\|\nabla u^{\varepsilon}\right\|_{L^{2}\left(\Omega^{\varepsilon}\right)},
$$

where the constants $C_{1}, C_{2}$ are independent of $\varepsilon$.

From (42), (43) and the uniform boundedness of the norm $\left\|f^{\varepsilon}\right\|_{L^{2}\left(\Omega^{\varepsilon}\right)}$ with respect to $\varepsilon$, it follows that the sequence of the functions $\left\{\widetilde{u}^{\varepsilon}(x)\right\}_{\varepsilon}$ is uniformly bounded and weakly compact in $\stackrel{\circ}{H}^{1}(\Omega)$ with respect to $\varepsilon$. Hence, we can extract a subsequence $\left\{\widetilde{u}^{\varepsilon_{m}}(x), m=1, \ldots, \infty\right\}$ that is weakly convergent in $\stackrel{\circ}{H}^{1}(\Omega)$ and strongly convergent in $L^{p}(\Omega)$ to some function $u(x) \in \stackrel{\circ}{H}^{1}(\Omega)$.

Now we will show that the function $u(x)$ is a minimizer of (15).

5.2. Proof of inequality (40). Let $\left\{\varepsilon_{k}\right\}_{k=1}^{\infty}, \quad\left\{h_{k}^{\prime}\right\}_{k=1}^{\infty}, \quad\left\{h_{k}^{\prime \prime}=h_{k}^{\prime}+\right.$ $\left.\left(h_{k}^{\prime}\right)^{1+\tau / 2}\right\}_{k=1}^{\infty}$ be the sequences constructed in Lemma 2. By assertion 3) of this lemma, we construct a sequence of sets $\Omega_{\mu_{1}} \subset \Omega_{\mu_{2}} \subset \cdots \subset \Omega_{\mu_{k}} \subset \cdots \subset \Omega$ such that mes $\left\{\Omega \backslash \Omega_{\mu_{k}}\right\}<\mu_{k}$. Setting $\mu_{k}=h_{k}^{\prime \prime}$, we get $\operatorname{mes}\left\{\Omega \backslash \Omega_{\mu_{k}}\right\}<h_{k}^{\prime \prime}$.

As in Lemma 6 , we cover the domain $\Omega$ by disjoint cubes $K_{h_{k}}^{\alpha}\left(\Omega \in \cup_{\alpha} K_{h_{k}}^{\alpha}\right)$ of the size $h_{k}=\frac{h_{k}^{\prime \prime}+h_{k}^{\prime}}{2}$ centered at the points $x^{\alpha}$. Taking into account that mes $s\left\{\Omega \backslash \Omega_{\mu_{k}}\right\}<h_{k}^{\prime \prime}$ and the total number of cubes $N_{k} \sim \frac{\operatorname{mes} s\{\Omega\}}{h^{n}}$, we get the estimate for the number of cubes with centers $x^{\alpha} \notin \Omega_{\mu_{k}}$ :

$$
N_{k}^{\prime}=O\left(\left(h_{k}\right)^{1-n}\right) .
$$

We also consider the cubes $K_{h_{k}^{\prime \prime}}^{\alpha}$ and $K_{h_{k}^{\prime}}^{\alpha}$ centered at $x^{\alpha}$.

We denote

$$
\begin{gathered}
\Lambda_{k}:=\left\{\alpha \in\left\{1, \ldots, N_{k}\right\}: x^{\alpha} \in \Omega_{\mu_{k}}, K_{k}^{\alpha} \in \Omega\right\}, \\
\Omega^{k}:=\Omega^{\varepsilon_{k}}, F^{k}:=F^{\varepsilon_{k}}, K_{k}^{\alpha}:=K_{h_{k}^{\prime \prime}}^{\alpha}, \Pi_{k}^{\alpha}:=K_{h_{k}^{\prime \prime}}^{\alpha} \backslash K_{h_{k}^{\prime}}^{\alpha}, \\
\Phi^{k}[u]:=\Phi^{\varepsilon_{k}}[u], g^{k}(x, u):=g^{\varepsilon_{k}}(x, u), \sigma^{k}(x, u):=\sigma^{\varepsilon_{k}}(x, u), f^{k}(x):=f^{\varepsilon_{k}}(x) .
\end{gathered}
$$


Let $w(x)$ be an arbitrary function from $C_{0}^{2}(\Omega), \widehat{w}_{k}^{\alpha}$ and $B_{k}^{\alpha}$ be the functions and sets constructed in Lemma 5 with $\widehat{s}=w\left(x^{\alpha}\right)$ for the cubes $K_{k}^{\alpha}$; $\widehat{v}_{k i}(i=$ $1, \ldots, n)$, and $\widehat{B}_{k}$ be the functions and sets constructed in Lemma 7 for the set $B_{k}=\cup_{\alpha} B_{k}^{\alpha} ;\left\{\varphi_{k}^{\alpha}(x)\right\}_{\alpha}$ be a partition of unity (28) associated with the covering of $\Omega$ by the cubes $K_{h_{k}^{\prime}}^{\alpha}$ and $K_{h_{k}^{\prime \prime}}^{\alpha}$. Consider the function

$$
w_{k}(x)=w(x)+\sum_{i=1}^{n} \frac{\partial w(x)}{\partial x_{i}}\left[\widehat{v}_{k i}(x)-x_{i}\right]+\sum_{\alpha}\left[\widehat{w}_{k}^{\alpha}(x)-w\left(x^{\alpha}\right)\right] \varphi_{k}^{\alpha}(x) .
$$

Here we set $\widehat{w}_{k}^{\alpha}(x)=w\left(x^{\alpha}\right)$ if $\alpha \notin \Lambda_{k}$. It is obvious that $w_{k}(x) \in H^{1}\left(\Omega^{k}, \partial \Omega\right)$.

We substitute the function $w_{k}(x)$ into functional (15) and estimate each term.

We estimate the first term. By (45), we can write the derivatives of $w_{k}(x)$ in the form

$$
\frac{\partial w_{k}}{\partial x_{j}}=\sum_{i=1}^{n} \frac{\partial w}{\partial x_{i}} \frac{\partial \widehat{v}_{k i}}{\partial x_{j}}+\sum_{\alpha \in \Lambda_{k}} \frac{\partial \widehat{w}_{k}^{\alpha}}{\partial x_{j}} \varphi_{k}^{\alpha}(x)+\sum_{s=1}^{2} A_{s}(x),
$$

where

$$
A_{1}(x)=\sum_{i=1}^{n} \frac{\partial^{2} w}{\partial x_{i} \partial x_{j}}\left[\widehat{v}_{k i}(x)-x_{i}\right], A_{2}(x)=\sum_{\alpha \in \Lambda_{k}}\left[\widehat{w}_{k}^{\alpha}(x)-w\left(x^{\alpha}\right)\right] \frac{\partial \varphi_{k}^{\alpha}(x)}{\partial x_{j}} .
$$

The selected terms in (46) give a finite contribution to the functional $\Phi^{k}\left[w_{k}\right]$, the contribution of the terms $A_{1}(x), A_{2}(x)$ vanishes. Indeed,

$$
\begin{aligned}
\int_{\Omega^{k}}\left|\nabla w_{k}\right|^{2} d x \leqslant & \sum_{i, j=1}^{n} \int_{\Omega^{k}}\left(\nabla \widehat{v}_{k i}, \nabla \widehat{v}_{k j}\right) \frac{\partial w}{\partial x_{i}} \frac{\partial w}{\partial x_{j}} d x \\
& +\sum_{\alpha \in \Lambda_{k}} \int_{K_{k}^{\alpha} \cap \Omega^{k}}\left|\nabla \widehat{w}_{k}^{\alpha}\right|^{2} d x+\sum_{\alpha \in \Lambda_{k}} E_{k}^{\alpha} .
\end{aligned}
$$

Here, $E_{k}^{\alpha}$ denotes the sum (over the sets $\Pi_{k}^{\alpha} \cap \Omega^{k}$ and $K_{k}^{\alpha} \cap B_{k}^{\alpha}$ ) of integrals of quadratic and linear combinations of the function $\left(\widehat{v}_{k i}-x_{i}\right)$ and $\left[\widehat{w}_{k}^{\alpha}(x)-w\left(x^{\alpha}\right)\right]$. $\frac{\partial \varphi_{k}^{\alpha}}{\partial x_{j}}$ with bounded coefficients that are equal to 1 or depend on $w \in C_{0}^{2}(\Omega)$ for the quadratic terms, and on $\frac{\partial \widehat{w}_{k}^{\alpha}}{\partial x_{j}} t \varphi_{k}^{\alpha}$ or $\frac{\partial \widehat{v}_{k i}}{\partial x_{j}}$ for the linear terms. We use the properties of the functions $\varphi_{k}^{\alpha}$ and Lemmas 5 and 7 to estimate $\sum E_{k}^{\alpha}$. As a result, we get

$$
\lim _{k \rightarrow \infty} \sum_{\alpha \in \Lambda_{k}} E_{k}^{\alpha}=0
$$

We can now proceed to estimate the surface integral in the functional $\Phi^{k}\left[w_{k}\right]$. To this end, we write $w_{k}(x)$ in the form

$$
w_{k}(x)=\sum_{\alpha \in \Lambda_{k}}\left[\widehat{w}_{k}^{\alpha}(x)+\left(w(x)-w\left(x^{\alpha}\right)\right)\right] \varphi_{k}^{\alpha}(x)
$$




$$
+\sum_{\alpha \notin \Lambda_{k}} w(x) \varphi_{k}^{\alpha}(x)+\sum_{i=1}^{n} \frac{\partial w(x)}{\partial x_{i}}\left[\widehat{v}_{k i}(x)-x_{i}\right] .
$$

For the second term, by taking into account (13), the properties $a_{1}-a_{3}$ of the function $\sigma^{k}(x, u)$, estimate 2) of Lemma 7, and (44), we obtain

$$
\begin{aligned}
\int_{\partial F^{k}} g^{k}\left(x, w_{k}\right) d \Gamma= & \sum_{\alpha \in \Lambda_{k}} \int_{K_{k}^{\alpha} \cap \partial F^{k}} g^{k}\left(x, w_{k}\right) d \Gamma+\sum_{\alpha \notin \Lambda_{k}} \int_{K_{k}^{\alpha} \cap \partial F^{k}} g^{k}\left(x, w_{k}\right) d \Gamma \\
= & \sum_{\alpha \in \Lambda_{k}} \int_{K_{k}^{\alpha} \cap \partial F^{k}} g^{k}\left(x, \widehat{w}_{k}^{\alpha}\right) d \Gamma \\
& \quad+\sum_{\alpha \notin \Lambda_{k}} \int_{K_{k}^{\alpha} \cap \partial F^{k}} g^{k}(x, w) d \Gamma+O\left(h_{k}^{\prime \prime}\right) \\
= & \sum_{\alpha \in \Lambda_{k}} \int_{K_{k}^{\alpha} \cap \partial F^{k}} g^{k}\left(x, \widehat{w}_{k}^{\alpha}\right) d \Gamma+O\left(h_{k}^{\prime \prime}\right)+C\left(\varepsilon_{k}\right),
\end{aligned}
$$

where $C\left(\varepsilon_{k}\right) \rightarrow 0$ as $k \rightarrow \infty$. Thus,

$$
\int_{\partial F^{k}} g^{k}\left(x, w_{k}\right) d \Gamma=\sum_{\alpha \in \Lambda_{k}} \int_{K_{k}^{\alpha} \cap \partial F^{k}} g^{k}\left(x, \widehat{w}_{k}^{\alpha}\right) d \Gamma+o(1), \quad k \rightarrow \infty .
$$

Consider now the third term in $\Phi^{k}\left[w_{k}\right]$. By (45), condition 3) of Theorem 2, and the estimates from Lemmas 5 and 7 , we obtain

$$
\int_{\Omega^{k}} f^{k}(x) w_{k}(x) d x=\int_{\Omega^{k}} f^{k}(x) w(x) d x+o(1), \quad k \rightarrow \infty .
$$

Thus, by virtue of $(47)-(50)$, the functional $\Phi^{k}\left[w_{k}\right]$ can be estimated as follows:

$$
\begin{aligned}
& \Phi^{k}\left[w_{k}\right]=\sum_{i, j=1}^{n} \int_{\Omega^{k}}\left(\nabla \widehat{v}_{k i}, \nabla \widehat{v}_{k j}\right) \frac{\partial w}{\partial x_{i}} \frac{\partial w}{\partial x_{j}} d x-2 \int_{\Omega^{k}} f^{k} w d x \\
& +\sum_{\alpha \in \Lambda_{k}}\left[\int_{K_{k}^{\alpha} \cap \Omega^{k}}\left|\nabla \widehat{w}_{k}^{\alpha}\right|^{2} d x+\int_{K_{k}^{\alpha} \cap \partial F^{k}} g^{k}\left(x, \widehat{w}_{k}^{\alpha}\right) d \Gamma\right]+o(1), \quad k \rightarrow \infty .
\end{aligned}
$$

Passing to the limit as $k \rightarrow \infty$ and taking into account the conditions of Theorem 2 and the estimates from Lemmas 5 and 7 , we obtain

$$
\varlimsup_{k \rightarrow \infty} \Phi^{k}\left[w_{k}\right] \leqslant \int_{\Omega} \sum_{i, j=1}^{n} a_{i j}(x) \frac{\partial w}{\partial x_{i}} \frac{\partial w}{\partial x_{k}} d x
$$




$$
+\int_{\Omega} c(x, w) d x-2 \int_{\Omega} f(x) w(x) d x .
$$

Since the function $u^{\varepsilon}$ minimizes the functional $\Phi^{\varepsilon}[\cdot]$ and from any sequence $\varepsilon \rightarrow 0$ we can choose the subsequence $\left\{\varepsilon=\varepsilon_{k} \rightarrow 0, k=1, \ldots, \infty\right\}$, for which inequality (51) is true, then

$$
\varlimsup_{\varepsilon \rightarrow 0} \Phi^{\varepsilon}\left[u^{\varepsilon}\right] \leqslant \varlimsup_{k \rightarrow \infty} \Phi^{k}\left[w_{k}\right] \leqslant \Phi[w],
$$

where $\Phi[w]$ is the energy functional of homogenized problem (2) defined by formula (37).

Hence, $\forall w(x) \in C_{0}^{2}(\Omega)$, inequality (40) is true. Since $C_{0}^{2}(\Omega)$ is dense in $\stackrel{\circ}{H}^{1}(\Omega)$, this inequality is true $\forall w(x) \in \stackrel{\circ}{H}^{1}(\Omega)$.

5.3. Proof of inequality (41). Let $u(x)$ be the weak limit in $H^{1}(\Omega)$ of the extended solutions $\widetilde{u}^{\varepsilon}(x)$ of problem (1) on some subsequence $\left\{\varepsilon=\varepsilon_{m}, m=\right.$ $1, \ldots, \infty\}$. From the embedding theorems it follows that the traces of $\widetilde{u}^{\varepsilon_{m}}(x)$ and $u(x)$ on $\partial \Omega$ are preserved and equal to 0 . Thus the function $u(x) \in \stackrel{\circ}{H}^{1}(\Omega)$.

As before, for the sake of brevity, we introduce the following notation:

$$
\begin{array}{lll}
u_{m}(x):=u^{\varepsilon_{m}}(x), & \widetilde{u}_{m}(x):=\widetilde{u}^{\varepsilon_{m}}(x), & \Omega^{m}:=\Omega^{\varepsilon_{m}}, \\
F^{m}:=F^{\varepsilon_{m}}, & \Phi^{m}[u]:=\Phi^{\varepsilon_{m}}[u], & g^{m}(x, u):=g^{\varepsilon_{m}}(x, u), \\
\sigma^{m}(x, u):=\sigma^{\varepsilon_{m}}(x, u), & \widehat{\sigma}^{m}(x):=\widehat{\sigma}^{\varepsilon_{m}}(x), & f^{m}(x):=f^{\varepsilon_{m}}(x) .
\end{array}
$$

Let $u^{\delta}(x) \in C_{0}^{2}(\Omega)$ be an approximation of $u(x)$ such that

$$
\left\|u^{\delta}-u\right\|_{H^{1}(\Omega)}<\delta .
$$

In the domains $\Omega^{m}$, we consider the functions

$$
\begin{aligned}
& \widetilde{u}_{m}^{\delta}(x)=\widetilde{u}_{m}(x)+u^{\delta}(x)-u(x) \in \stackrel{\circ}{H}^{1}(\Omega), \\
& u_{m}^{\delta}(x)=\left.\widetilde{u}_{m}^{\delta}(x)\right|_{\Omega^{m}}=u_{m}(x)+u^{\delta}(x)-u(x) \in H^{1}\left(\Omega^{m}, \partial \Omega\right) .
\end{aligned}
$$

By (52), we have

$$
\left\|\widetilde{u}_{m}^{\delta}-\widetilde{u}_{m}\right\|_{H^{1}(\Omega)}<\delta, \quad\left\|u_{m}^{\delta}-u_{m}\right\|_{H^{1}\left(\Omega^{m}\right)}<\delta,
$$

in addition, as $m \rightarrow \infty$,

$$
\left\|\widetilde{u}_{m}^{\delta}-u^{\delta}\right\|_{L^{2}(\Omega)} \rightarrow 0, \quad\left\|u_{m}^{\delta}-u^{\delta}\right\|_{L^{2}\left(\Omega^{m}, \Omega\right)} \rightarrow 0 .
$$

We define the functions

$$
v_{m}^{\delta}(x)=\widetilde{u}_{m}^{\delta}(x)-u^{\delta}(x) \in \stackrel{\circ}{H}^{1}(\Omega) .
$$


Since, the functions $v_{m}^{\delta}$ converge strongly to 0 in $L^{2}(\Omega)$ and weakly to 0 in $H^{1}(\Omega)$ as $m \rightarrow \infty$, we conclude that $\left\|v_{m}^{\delta}\right\|_{H^{1}(\Omega)} \leqslant C$ and the functions $v_{m}^{\delta}(x)$ converge in measure to 0 . Namely, there exist the sets $G^{m}:=G^{\varepsilon_{m}} \subset \Omega$ and the numbers $\beta_{m}:=\beta\left(\varepsilon_{m}\right)$ such that

$$
\forall x \in \Omega \backslash G^{m}:\left|v_{m}^{\delta}(x)\right|<\beta_{m}, \lim _{m \rightarrow \infty} \beta_{m}=0, \lim _{m \rightarrow \infty} \operatorname{mes}\left\{G^{m}\right\}=0 .
$$

By virtue of Lemma 1.4 [12, Chap. 3], on the bases of the functions $v_{m}^{\delta}$ and the sets $G^{m}$, we can construct the functions $\widehat{v}_{m}^{\delta} \in \stackrel{\circ}{H}^{1}(\Omega)$ and the sets $\widehat{G}^{m} \subset \Omega$ such that $\widehat{G}^{m} \supset G^{m}, \widehat{v}_{m}^{\delta}=v_{m}^{\delta}$ for $x \in \Omega \backslash \widehat{G}^{m}$, and

$$
\max _{\Omega}\left|\widehat{v}_{m}^{\delta}(x)\right| \leqslant C \beta_{m}, \lim _{m \rightarrow \infty}\left\|\widehat{v}_{m}^{\delta}\right\|_{H^{1}\left(\widehat{G}^{m}\right)}=0, \lim _{m \rightarrow \infty} \operatorname{mes} s\left\{\widehat{G}^{m}\right\}=0 .
$$

Using these functions, we define the functions

$$
\widehat{u}_{m}^{\delta}(x)=u^{\delta}(x)+\widehat{v}_{m}^{\delta}(x) \in \stackrel{\circ}{H}^{1}(\Omega) .
$$

Let us cover $\Omega$ with disjoint cubes $K_{m}^{\alpha}=K\left(x^{\alpha}, h_{m}\right)$ of the size $h_{m}$ centered at $x^{\alpha}$. The centers $x^{\alpha}$ and the sizes $h_{m}$ are chosen as follows: $h_{m}$ is defined as a minimum value for which the following inequalities are true:

$$
\begin{aligned}
& \max _{\Omega}\left|\widehat{v}_{m}^{\delta}(x)\right|<h_{m}, \quad\left\|\widehat{u}_{m}^{\delta}-u^{\delta}\right\|_{L^{2}(\Omega)}<h_{m}^{2}, \\
& \operatorname{mes}\left\{\widehat{G}^{m}\right\}<h_{m}^{2+\tau_{1}}, \quad\left\|\widehat{v}_{m}^{\delta}\right\|_{H^{1}\left(\widehat{G}^{m}\right)}<h_{m}^{2+\tau_{1}}, \quad \tau_{1}>\tau>0 .
\end{aligned}
$$

By Lemma 2, we construct a sequence of sets $\Omega_{\mu_{1}} \subset \Omega_{\mu_{2}} \subset \ldots \subset \Omega_{\mu_{m}} \subset \ldots \subset \Omega$ such that mes $\left\{\Omega \backslash \Omega_{\mu_{m}}\right\}<h_{m}$. We choose the centers $x^{\alpha}$ of the cubes $K_{m}^{\alpha}$ as in Lemma 6. According to the assertions of Lemma 2, the equalities

$$
\begin{aligned}
& \lim _{m \rightarrow \infty} \frac{1}{h_{m}^{n}}\left|c\left(x^{\alpha}, s ; \varepsilon_{m}, h_{m}\right)-c\left(x^{\alpha}, s\right) \cdot h_{m}^{n}\right|=0, \\
& \lim _{m \rightarrow \infty} \frac{1}{h_{m}^{n}}\left|a_{i j}\left(x^{\alpha}, \varepsilon_{m}, h_{m}\right)-a_{i j}\left(x^{\alpha}\right) \cdot h_{m}^{n}\right|=0, \quad i, j=1, \ldots, n
\end{aligned}
$$

hold uniformly with respect to $x^{\alpha} \in \Omega_{\mu_{m}}$.

We denote

$$
\Lambda_{m}:=\left\{\alpha \in\left\{1, \ldots, N_{m}\right\}: x^{\alpha} \in \Omega_{\mu_{m}}, K_{m}^{\alpha} \in \Omega\right\} .
$$

In the intersections $K_{m}^{\alpha} \cap \Omega^{m}$, we consider the functions

$$
w_{m 1}^{\delta \alpha}(x)=\widehat{u}_{m}^{\delta}(x)-u^{\delta}\left(x^{\alpha}\right) .
$$

Since $u^{\delta}(x) \in C_{0}^{2}(\Omega)$, the inequality 


$$
\begin{aligned}
\int_{K_{m}^{\alpha} \cap \Omega^{m}} \mid w_{m 1}^{\delta \alpha}(x) & -\left.\left(x-x^{\alpha}, \ell\right)\right|^{2} d x \leqslant 3 \int_{K_{m}^{\alpha} \cap \Omega^{m}}\left|\widehat{u}_{m}^{\delta}(x)-u^{\delta}(x)\right|^{2} d x \\
& +3 \int_{K_{m}^{\alpha} \cap \Omega^{m}}\left[\left(\nabla u^{\delta}\left(x^{\alpha}\right), x-x^{\alpha}\right)-\left(x-x^{\alpha}, \ell\right)\right]^{2} d x+O\left(h_{m}^{n+4}\right)
\end{aligned}
$$

holds for all $\ell \in R^{n}$ and any fixed number $\delta>0$. Setting $\ell=\nabla u^{\delta}\left(x^{\alpha}\right)$ and taking into account (56), we obtain

$$
\int_{K_{m}^{\alpha} \cap \Omega^{m}}\left|w_{m 1}^{\delta \alpha}(x)-\left(\nabla u^{\delta}\left(x^{\alpha}\right), x-x^{\alpha}\right)\right|^{2} d x=O\left(h_{m}^{n+4}\right) .
$$

By virtue of definition (8) of the functional $T_{h, z}^{\varepsilon}(\ell)$ and its presentation (10) for $\ell=\nabla u^{\delta}\left(x^{\alpha}\right)$, we have

$$
\begin{aligned}
\int_{K_{m}^{\alpha} \cap \Omega^{m}}\left|\nabla w_{m 1}^{\delta \alpha}(x)\right|^{2}+h^{-2-\tau}\left|w_{m 1}^{\delta \alpha}(x)-\left(\nabla u^{\delta}\left(x^{\alpha}\right), x-x^{\alpha}\right)\right|^{2} d x \\
\geqslant \sum_{i, j=1}^{n} a_{i j}\left(x^{\alpha}, \varepsilon_{m}, h_{m}\right) \frac{\partial u^{\delta}}{\partial x_{i}}\left(x^{\alpha}\right) \frac{\partial u^{\delta}}{\partial x_{j}}\left(x^{\alpha}\right) .
\end{aligned}
$$

From (55), (56), (58)-(60), taking into account that $\alpha=1, \ldots, N_{m} \sim \frac{\operatorname{mes}\{\Omega\}}{h_{m}^{n}}$, $u_{m}^{\delta}(x)=\widehat{u}_{m}^{\delta}(x)$ for $x \in \Omega^{m} \backslash \widehat{G}^{m}$ and $\nabla w_{m 1}^{\delta \alpha}(x)=\nabla \widehat{u}_{m}^{\delta}(x)$ for $x \in \Omega^{m}$, we obtain

$$
\begin{aligned}
\sum_{\alpha} \int_{K_{m}^{\alpha} \cap \Omega^{m} \backslash \widehat{G}^{m}}\left|\nabla u_{m}^{\delta}(x)\right|^{2} d x=\sum_{\alpha} \int_{K_{m}^{\alpha} \cap \Omega^{m} \backslash \widehat{G}^{m}}\left|\nabla \widehat{u}_{m}^{\delta}(x)\right|^{2} d x \\
\geqslant \sum_{\alpha} \sum_{i, j=1}^{n} a_{i j}\left(x^{\alpha}, \varepsilon_{m}, h_{m}\right) \frac{\partial u^{\delta}}{\partial x_{i}}\left(x^{\alpha}\right) \frac{\partial u^{\delta}}{\partial x_{j}}\left(x^{\alpha}\right) \\
-\int_{\Omega^{m} \cap \widehat{G}^{m}}\left|\nabla \widehat{u}_{m}^{\delta}(x)\right|^{2} d x-O\left(h_{m}^{2-\tau}\right) \\
=\sum_{\alpha} \sum_{i, j=1}^{n} a_{i j}\left(x^{\alpha}, \varepsilon_{m}, h_{m}\right) \frac{\partial u^{\delta}}{\partial x_{i}}\left(x^{\alpha}\right) \frac{\partial u^{\delta}}{\partial x_{j}}\left(x^{\alpha}\right)-O\left(h_{m}^{2-\tau}\right) .
\end{aligned}
$$

Thus,

$$
\begin{aligned}
\sum_{\alpha} \int_{K_{m}^{\alpha} \cap \Omega^{m} \backslash \widehat{G}^{m}}\left|\nabla u_{m}^{\delta}(x)\right|^{2} d x & \\
& \geqslant \sum_{\alpha} \sum_{i, j=1}^{n} a_{i j}\left(x^{\alpha}, \varepsilon_{m}, h_{m}\right) \frac{\partial u^{\delta}}{\partial x_{i}}\left(x^{\alpha}\right) \frac{\partial u^{\delta}}{\partial x_{j}}\left(x^{\alpha}\right)-O\left(h_{m}^{2-\tau}\right) .
\end{aligned}
$$


Further, in the intersections $K_{m}^{\alpha} \cap \Omega^{m}$, we consider the functions

$$
w_{m 2}^{\delta \alpha}(x)=u^{\delta}\left(x^{\alpha}\right)+u_{m}^{\delta}(x)-\widehat{u}_{m}^{\delta}(x) .
$$

By virtue of definition (12) of the functional $c(x, s ; \varepsilon, h)$, we have

$$
\begin{aligned}
\sum_{\alpha} c\left(x^{\alpha}, u^{\delta}\left(x^{\alpha}\right) ; \varepsilon_{m}, h_{m}\right) \leqslant \sum_{\alpha} & \int_{K_{m}^{\alpha} \cap \Omega^{m}}\left|\nabla w_{m 2}^{\delta \alpha}\right|^{2} d x \\
& +h_{m}^{-\tau-2} \sum_{\alpha} \int_{K_{m}^{\alpha} \cap \Omega^{m}}\left|w_{m 2}^{\delta \alpha}(x)-u^{\delta}\left(x^{\alpha}\right)\right|^{2} d x \\
& +\sum_{\alpha} \int_{K_{m}^{\alpha} \cap \partial F^{m}} g^{m}\left(x, w_{m 2}^{\delta \alpha}\right) d \Gamma
\end{aligned}
$$

We now consider each term of the right-hand side. By the Minkowski inequality, the definitions of $w_{m 2}^{\delta \alpha}(62)$ and of $\widehat{u}_{m}^{\delta}(x)$ (55) and (56) for the first and second terms, we obtain

$$
\begin{aligned}
\sum_{\alpha} \int_{K_{m}^{\alpha} \cap \Omega^{m}}\left|\nabla w_{m 2}^{\delta \alpha}\right|^{2} d x & =\int_{\Omega^{m} \cap \widehat{G}^{m}}\left|\nabla w_{m 2}^{\delta \alpha}\right|^{2} d x \\
& =\int_{\Omega^{m} \cap \widehat{G}^{m}}\left|\nabla\left(u_{m}^{\delta}-u^{\delta}-\widehat{v}_{m}^{\delta}\right)\right|^{2} d x \\
& \leqslant\left(\sqrt{\int_{\Omega^{m} \cap \widehat{G}^{m}}\left|\nabla u_{m}^{\delta}\right|^{2} d x}+O\left(h_{m}^{1+\tau_{1} / 2}\right)\right)^{2} \\
& =\int_{\Omega^{m} \cap \widehat{G}^{m}}\left|\nabla u_{m}^{\delta}\right|^{2} d x+O\left(h_{m}^{1+\tau_{1} / 2}\right) \\
\sum_{\alpha} \int_{K_{m}^{\alpha} \cap \Omega^{m}}\left|w_{m 2}^{\delta \alpha}(x)-u^{\delta}\left(x^{\alpha}\right)\right|^{2} d x & \\
& =\int_{\Omega^{m} \cap \widehat{G}^{m}}\left|u_{m}^{\delta}(x)-\widehat{u}_{m}^{\delta}(x)\right|^{2} d x=O\left(h_{m}^{2+\tau_{1}}\right) .
\end{aligned}
$$

Let us estimate the surface integral. By the smoothness of the function $u^{\delta}(x)$ and (56), we write $w_{m 2}^{\delta \alpha}=u_{m}^{\delta}(x)-\left(u^{\delta}(x)-u^{\delta}\left(x^{\alpha}\right)\right)-\widehat{v}_{m}^{\delta}(x)=u_{m}^{\delta}(x)+O\left(h_{m}\right)$ for $x \in \Omega^{m} \cap K_{m}^{\alpha}$. By the properties $a_{1}-a_{3}$ of the function $\sigma^{m}(x, u)$,

$$
\begin{aligned}
\sum_{\alpha} \int_{K_{m}^{\alpha} \cap \partial F^{m}} g^{m}\left(x, w_{m 2}^{\delta \alpha}\right) d \Gamma & =\int_{\partial F^{m}} g^{m}\left(x, u_{m}^{\delta}\right) d \Gamma \\
+ & O\left(h_{m}\right) \int_{\partial F^{m}}\left|u_{m}^{\delta}\right|^{\Theta} \widehat{\sigma}^{m}(x) d \Gamma+O\left(h_{m}\right) \\
& =\int_{\partial F^{m}} g^{m}\left(x, u_{m}^{\delta}\right) d \Gamma+O\left(h_{m}\right)\left\|u_{m}^{\delta}\right\|_{L^{\Theta}\left(\Omega, \mu^{m}\right)}^{\Theta}+O\left(h_{m}\right) .
\end{aligned}
$$

Journal of Mathematical Physics, Analysis, Geometry, 2017, Vol. 13, No. 3 
Here $L^{\Theta}\left(\Omega, \mu^{m}\right)$ is a space with measure $d \mu^{m}=\widehat{\sigma}^{m}(x) d \Gamma, \Theta<\frac{n}{n-2}$. By the property $a_{3}$ of the function $\sigma^{m}(x, u)$, we have the inequality

$$
\int_{\partial F^{m} \cap B(\rho, z)} d \mu^{m}<C_{1} \rho^{n}+C_{2}\left(\varepsilon_{m}\right) \rho^{n-1}
$$

where $C_{2}\left(\varepsilon_{m}\right) \rightarrow 0$ as $m \rightarrow \infty$. The embedding of the space $H^{1}(\Omega)$ into $L^{\Theta}\left(\Omega, \mu^{m}\right)$ follows from Sobolev's generalized theorem ( $[13$, p. 58$\left.]\right)$. Under the condition $H^{1}(\Omega) \subset L^{\Theta}\left(\Omega, \mu^{m}\right)$, (53) and the fact that the sequence of extended solutions of problem (1) is uniformly bounded in $H^{1}(\Omega)$, the functions $u_{m}^{\delta}$ and $\widetilde{u}_{m}^{\delta}$ satisfy the inequalities

$$
\begin{aligned}
\left\|u_{m}^{\delta}\right\|_{L^{\Theta}\left(\Omega, \mu^{m}\right)} & =\left\|\widetilde{u}_{m}^{\delta}\right\|_{L^{\Theta}\left(\Omega, \mu^{m}\right)} \leqslant C_{1}\left\|\widetilde{u}_{m}^{\delta}\right\|_{H^{1}(\Omega)} \\
& \leqslant C_{1}\left(\left\|\widetilde{u}_{m}\right\|_{H^{1}(\Omega)}+\delta\right) \leqslant C_{2} .
\end{aligned}
$$

Thus, for the surface integral, the estimate

$$
\sum_{\alpha} \int_{K_{m}^{\alpha} \cap \partial F^{m}} g^{m}\left(x, w_{m 2}^{\delta \alpha}\right) d \Gamma=\int_{\partial F^{m}} g^{m}\left(x, u_{m}^{\delta}\right) d \Gamma+O\left(h_{m}\right)
$$

holds.

By (64)-(67), from (63) it follows

$$
\begin{aligned}
\sum_{\alpha} c\left(x^{\alpha}, u^{\delta}\left(x^{\alpha}\right) ; \varepsilon_{m}, h_{m}\right) \leqslant & \int_{\Omega^{m} \cap \widehat{G}^{m}}\left|\nabla u_{m}^{\delta}\right|^{2} d x \\
& \quad+\int_{\partial F^{m}} g^{m}\left(x, u_{m}^{\delta}\right) d \Gamma+O\left(h_{m}^{\tau_{1}-\tau}\right) .
\end{aligned}
$$

Hence, by virtue of the obtained estimates (61), (68) and the positivity of summable functions, we have

$$
\begin{aligned}
\Phi^{m}\left[u_{m}^{\delta}\right]= & \sum_{\alpha} \int_{K_{m}^{\alpha} \cap \Omega^{m} \backslash \widehat{G}^{m}}\left|\nabla u_{m}^{\delta}\right|^{2} d x+\int_{\Omega^{m} \cap \widehat{G}^{m}}\left|\nabla u_{m}^{\delta}\right|^{2} d x \\
& +\int_{\partial F^{m}} g^{m}\left(x, u_{m}^{\delta}\right) d \Gamma-2 \int_{\Omega^{m}} f^{m} u_{m}^{\delta} d x \\
\geqslant & \sum_{\alpha \in \Lambda_{m}} \sum_{i, j=1}^{n} a_{i j}\left(x^{\alpha}, \varepsilon_{m}, h_{m}\right) \frac{\partial u^{\delta}}{\partial x_{i}}\left(x^{\alpha}\right) \frac{\partial u^{\delta}}{\partial x_{j}}\left(x^{\alpha}\right) \\
& +\sum_{\alpha \in \Lambda_{m}} c\left(x^{\alpha}, u^{\delta}\left(x^{\alpha}\right) ; \varepsilon_{m}, h_{m}\right)-2 \int_{\Omega^{m}} f^{m} u_{m}^{\delta} d x-O\left(h_{m}^{\tau_{1}-\tau}\right) .
\end{aligned}
$$

By (57), taking into account $\operatorname{mes}\left\{\Omega_{\mu_{m}}\right\} \rightarrow \operatorname{mes}\{\Omega\}$ as $m \rightarrow \infty$, the equalities

$$
\lim _{m \rightarrow \infty} \sum_{\alpha \in \Lambda_{m}} \sum_{i, j=1}^{n} a_{i j}\left(x^{\alpha}, \varepsilon_{m}, h_{m}\right) \frac{\partial u^{\delta}}{\partial x_{i}}\left(x^{\alpha}\right) \frac{\partial u^{\delta}}{\partial x_{j}}\left(x^{\alpha}\right)
$$




$$
\begin{aligned}
& =\lim _{m \rightarrow \infty} \int_{\Omega_{\mu_{m}}} \sum_{i, j=1}^{n} a_{i j}(x) \frac{\partial u^{\delta}}{\partial x_{i}} \frac{\partial u^{\delta}}{\partial x_{j}} d x \\
& =\int_{\Omega} \sum_{i, j=1}^{n} a_{i j}(x) \frac{\partial u^{\delta}}{\partial x_{i}} \frac{\partial u^{\delta}}{\partial x_{j}} d x \\
\lim _{m \rightarrow \infty} \sum_{\alpha \in \Lambda_{m}} c\left(x^{\alpha}, u^{\delta}\left(x^{\alpha}\right) ; \varepsilon_{m}, h_{m}\right) & =\lim _{m \rightarrow \infty} \int_{\Omega_{\mu_{m}}} c\left(x, u^{\delta}\right) d x=\int_{\Omega} c\left(x, u^{\delta}\right) d x .
\end{aligned}
$$

are true.

When passing to the limit as $m \rightarrow \infty$ for fixed $\delta$, we take into account the above equalities, condition 3) of Theorem 2 and (54), to get

$$
\begin{aligned}
\lim _{m \rightarrow \infty} \Phi^{m}\left[u_{m}^{\delta}\right] & \geqslant \int_{\Omega} \sum_{i, j=1}^{n} a_{i j}(x) \frac{\partial u^{\delta}}{\partial x_{i}} \frac{\partial u^{\delta}}{\partial x_{j}} d x+\int_{\Omega} c\left(x, u^{\delta}\right) d x-2 \int_{\Omega} f u^{\delta} d x \\
& =\Phi\left[u^{\delta}\right] .
\end{aligned}
$$

We now pass to the limit as $\delta \rightarrow 0$. In view of the smoothness of the function $u^{\delta}(x)$ and inequality (52), we get $\lim _{\delta \rightarrow 0} \Phi\left[u^{\delta}\right]=\Phi[u]$ on the right-hand side. On the left-hand side, passing to the limit in the surface integral, we use Sobolev's generalized theorem and inequality (52). As a result, we obtain the required inequality (41).

\subsection{Uniqueness of generalized solution of homogenized prob-} lem (2). In the proof we follow all steps as in the proof of Theorem 1. Assume that problem (2) has two generalized solutions $u_{1}, u_{2}$. Applying (14) for the functions $u_{1}, u_{2}$, subtracting one equality from another, and substituting $\varphi(x)=$ $u_{1}(x)-u_{2}(x)$ as a test function, we obtain

$$
\begin{aligned}
\int_{\Omega} \sum_{i, k=1}^{n} a_{i k}(x) \frac{\partial\left(u_{1}-u_{2}\right)}{\partial x_{k}} \frac{\partial\left(u_{1}-u_{2}\right)}{\partial x_{i}} d x & \\
& +\frac{1}{2} \int_{\Omega}\left(c_{u}\left(x, u_{1}\right)-c_{u}\left(x, u_{2}\right)\right)\left(u_{1}-u_{2}\right) d \Gamma=0 .
\end{aligned}
$$

From the above, by the positive definiteness of the tensor $\left\{a_{i k}(x)\right\}_{i, k=1}^{n}$ and monotonicity of the function $c_{u}(x, u)$, we get

$$
u_{1}=u_{2} \text { almost everywhere in } \Omega \text {. }
$$

Thus, the uniqueness of the generalized solution of homogenized problem (2) is proved. Theorem 2 is proved. 


\section{References}

[1] B. Cabarrubias and P. Donato, Homogenization of a Quasilinear Elliptic Problem with Nonlinear Robin Boundary Condition, Appl. Anal.: An Intern. J. 91 (2012), No. 6, 1111-1127.

[2] I. Chourabi and P. Donato, Homogenization of Elliptic Problems with Quadratic Growth and Nonhomogenous Robin Conditions in Perforated Domains, Chin. Ann. Math. 37B (2016), No. 6, 833-852.

[3] D. Cioranescu and P. Donato, On Robin Problems in Perforated Domains, Math. Sci. Appl. 9 (1997), 123-135.

[4] D. Cioranescu, P. Donato, and R. Zaki, Asymptotic Behaviour of Elliptic Problems in Perforated Domains with Nonlinear Boundary Conditions, Asymptot. Anal 53 (2007), 209-235.

[5] C. Conca, J. Diaz, A. Linan, and C. Timofte, Homogenization in Chemical Reactive Floes, Electron. J. Differential Equations 40 (2004), 1-22.

[6] C. Conca, J. Diaz, A. Linan, and C. Timofte, Homogenization Results for Chemical Reactive Flows Through Porous Media, New trends in continuum mechanics, Theta Ser. Adv. Math. 3, Theta, Bucharest, 2005, 99-107.

[7] C. Conca, J. Diaz, and C. Timofte, On the Homogenization of a Transmission Problem Arising in Chemistry, Romanian Rep. Phys. 56 (2004), No. 4, 613-622.

[8] L.C. Evans, Partial Differential Equations. Graduate Studies in Mathematics, Providence: Amer. Math. Soc., 1998.

[9] M.V. Goncharenko and L.A. Khilkova, Homogenized Model of Diffusion in Porous Media with Nonlinear Absorption at the Boundary, Ukrain. Mat. Zh. 67 (2015), No. 9, 1201-1216 (Russian); Engl. transl.: Ukrainian Math. J. 67 (2016), No. 9, $1349-1366$.

[10] M.V. Goncharenko and L.A. Khilkova, Homogenized Model of Diffusion in a LocallyPeriodic Porous Media with Nonlinear Absorption at the Boundary, Dopov. Nats. Akad. Nauk Ukr. 10 (2016), No. 6, 15-19 (Russian).

[11] A.N. Kolmogorov and S.V. Fomin, Elements of the Theory of Functions and Functional Analysis, Fizmatlit, Moscow, 2004 (Russian).

[12] V.A. Marchenko and E.Ya. Khruslov, Homogenization of Partial Differential Equations, Birkhöuser, Boston, Basel, Berlin, 2006.

[13] V.G. Maz'ya, Sobolev Spaces, Izdatel'stvo LGU, Leningrad, 1985 (Russian).

[14] T.A. Mel'nyk and O.A. Sivak, Asymptotic Analysis of a Boundary-Value Problem with the Nonlinean Multiphase Interactions in a Perforated Domain, Ukrain. Mat. Zh. 61 (2009), No. 4, 494-512.

[15] A.N. Tikhonov and A.A. Samarskiy, Equations of the Mathematical Physics, Nauka, Moscow, 1972 (Russian).

312 Journal of Mathematical Physics, Analysis, Geometry, 2017, Vol. 13, No. 3 
[16] C. Timofte, On the Homogenization of a Climatization Problem, Studia Univ. Babes-Bolyai Math LII (2007), No. 2, 117-125.

[17] C. Timofte, N. Cotfas, and G. Pavel, On the Asymptotic Behaviour of Some Elliptic Problems in Perforated Domains, Romanian Rep. Phys. 64 (2012), No. 1, 5-14. 